\title{
Phase Behaviour of 1-Ethyl-3-methylimidazolium Thiocyanate Ionic Liquid with Catalytic Deactivated Compounds and Water at Several Temperatures: Experiments and Theoretical Predictions
}

\author{
Ramalingam Anantharaj and Tamal Banerjee \\ Department of Chemical Engineering, Indian Institute of Technology Guwahati, Assam, Guwahati 781039, India \\ Correspondence should be addressed to Tamal Banerjee, tamalb@iitg.ernet.in
}

Received 16 March 2011; Revised 15 June 2011; Accepted 15 June 2011

Academic Editor: D. Yu. Murzin

Copyright ( 2011 R. Anantharaj and T. Banerjee. This is an open access article distributed under the Creative Commons Attribution License, which permits unrestricted use, distribution, and reproduction in any medium, provided the original work is properly cited.

\begin{abstract}
Density, surface tension and refractive index were determined for the binary mixture of catalytic deactivated compounds with 1-ethyl-3-methylimidazolium thiocyanate $\{[\mathrm{EMIM}][\mathrm{SCN}]\}$ at temperature of (298.15 to 323.15) K. For all the compounds with ILs, the densities varied linearly in the entire mole fraction with increasing temperature. From the obtained data, the excess molar volume and deviation of surface tension and refractive index have been calculated. A strong interaction was found between similar (cation-thiophene or cation-pyrrole) compounds. The interaction of IL with dissimilar compounds such as indoline and quinoline and other multiple ring compounds was found to strongly depend on the composition of IL at any temperatures. For the mixtures, the surface tension decreases in the order of: thiophene $>$ quinoline $>$ pyridine $>$ indoline $>$ pyrrole $>$ water. In general from the excess volume studies, the IL-sulphur/nitrogen mixture has stronger interaction as compared to IL-IL, thiophene-thiophene or pyrrole-pyrrole interaction. The deviation of surface tension was found to be inversely proportional to deviation of refractive index. The quantum chemical based COSMO-RS was used to predict the non-ideal liquid phase activity coefficient for all mixtures. It indicated an inverse relation between activity coefficient and excess molar volumes.
\end{abstract}

\section{Introduction}

Zero emission of nitrogen and sulphur in diesel oil production is one of the largest challenges for petroleum refineries in the world. However the hydrodenitrification (HDN) and hydrodesulphurization (HDS) processes represent an even more important challenge. Most of the refineries in the world are able to produce diesel oil with above $70 \mathrm{ppm}$ of nitrogen [1] and $500 \mathrm{ppm}$ of sulphur [2] by using high hydrogen consumption, large reactor volume, and severe operating conditions [3]. Thus the refinery must remove the aromatic compounds of nitrogen/sulphur such as pyrrole, indole, indoline, carbazole, benzocarbazole, pyridine, quinoline, benzoquinoline, thiophene, benzothiophene, dibenzothiophene, and their derivatives $[1,2,4]$. Moreover the catalysts having higher activity or selectivity do not interact favourably with such compounds, even at moderate temperatures [5]. On the other hand the nature of the compounds coupled with its chemical structure posseses more sterical hindrance as compared to the catalysts. The HDN and HDS processes have greater ability in removing nonaromatic structure of nitrogen and sulphur molecules from diesel oil with high efficiency although not economically $[6,7]$.

Over the past few years Ionic Liquid (ILs) are alternative solvent for organic solvents in various applications and are particularly used as an extracting solvent for the removal of sulphur from fuel oil [7-11]. The physiochemical properties of ILs are easily adjustable by changing cation and anion combination. Further it has negligible vapour pressure, posses nonflammability and high thermal and chemical stability. ILs provide better solution for removal of aromatic skeleton structure with multiple rings of nitrogen and sulphur compounds in diesel oil via $\mathrm{CH}-\pi$ bond interaction, $\pi-$ $\pi$ stacking, and $\mathrm{n}-\pi$ bond interaction [12-16]. Additionally ILs have higher LUMO/HOMO energy and high polarity in mixtures with such compounds [17].There are several 
works which report desulphurization using ionic liquid with a target of below $10 \mathrm{ppm}$ of total sulphur content in diesel and other liquid fuels. However these works are carried out without proper screening of ionic liquid (combinations as large as $10^{18}$ ILs!!).

In our previous work we have reported that smaller size of cation has higher selectivity but lower capacity for the removal of thiophene using COnductor-Like Screening MOdel for Real Solvent (COSMO-RS) predictions at $T=$ $298.15 \mathrm{~K}$ and atmospheric pressure [18]. Further we have studied the removal of pyrrole, indole, indoline, carbazole, benzocarbazole, pyridine, quinoline, benzoquinoline, thiophene, benzothiophene, dibenzothiophene, and their derivatives from diesel oil using smaller size cation based on imidazolium, pyridinium, pyrrolidinium, piperidinium, morpholinuim, and triazolium with fluoro- and nonfluoro anions $[13,14]$. According to these results we have chosen few ionic liquids for the removal of such compounds via Liquid-Liquid Extraction (LLE) at $T=298.15 \mathrm{~K}$. According to our work via COSMO-RS prediction [12-16] the IL: 1-ethyl-3-methylimidazolium thiocyanate ([EMIM][SCN]) was the most efficient IL for the removal of pyrrole, pyridine, indoline, quinoline, and thiophene molecules. Thus in this work we have studied the phase behaviour of [EMIM] [SCN] with such compounds at $p=1$ bar.

In a recent work, Domańska et al. [19] studied the phase behaviour of the binary system of thiophene with 1-ethyl3-methylimidazolium thiocyanate and other ionic liquids. In this work, they measured densities, surface tension, and refractive index of mixtures of [EMIM][SCN] with pyrrole, pyridine, indoline, quinoline, thiophene, and water. Further they have calculated the excess molar volume, deviation of surface tension, and refractive index of their mixture over the entire mole fraction of [EMIM] [SCN] at several temperature. The studied binary systems are $[$ EMIM] $[$ SCN $](1)+$ pyridine(2), [EMIM] $[$ SCN $](1)+$ pyrrole(2), [EMIM] [SCN](1)+quinoline(2), and [EMIM] [SCN](1)+ indoline(2), [EMIM][SCN](1) + thiophene(2) and $[\mathrm{EMIM}][\mathrm{SCN}](1)+$ water(2) over the 0.2 to 0.8 mole fraction of [EMIM] [SCN] (1) at $T=(298.15-323.15) \mathrm{K}$ and atmospheric pressure.

Finally the quantum chemical-based COSMO-RS (Conductor-like Screening MOdel for Real Solvents) [12-15] model has been used to predict the activity coefficient for known composition of mixtures. This model is independent of experimental data and uses the molecular structure to determine the Screening Charge Densities (SCDs) or the sigma profile of the pure molecule. This is the only indicator in computing the chemical potential of a component in the solution. The computational details of COSMO-RS will be described briefly in the later section.

\section{Experimental Section}

1-Ethyl-3-methylimidazolium thiocyanate [EMIM] [SCN]$\left(\mathrm{C}_{7} \mathrm{H}_{11} \mathrm{~N}_{3} \mathrm{~S}\right)$ ionic liquid was purchased from Fluka with a purity of $>95 \%$. In order to increase the purity and for reducing the water content and volatile compounds to negligible values, vacuum $(0.1 \mathrm{~Pa})$ for at least $48 \mathrm{~h}$ was applied to the IL sample prior to the measurements. A water content of $0.044 \%$ in [EMIM] [SCN] was obtained by Karl Fischer titrator (KF 787, Metrohm). Pyrrole $\left(\mathrm{C}_{4} \mathrm{H}_{5} \mathrm{~N}\right)$ and indoline $\left(\mathrm{C}_{8} \mathrm{H}_{9} \mathrm{~N}\right)$ were supplied from Spectrochem, India with mass fraction of 99\%. Pyridine $\left(\mathrm{C}_{5} \mathrm{H}_{5} \mathrm{~N}\right)$, quinoline $\left(\mathrm{C}_{9} \mathrm{H}_{7} \mathrm{~N}\right)$ and thiophene $\left(\mathrm{C}_{4} \mathrm{H}_{4} \mathrm{~S}\right)$ were received from Sigma-Aldrich with mass fractions greater than $98 \%$ and were used without further purification. The pure component structures, experimental densities, refractive index, and surface tension are given in Tables 1 to 3 . A comparison between reported and experimental measurements was carried out on all the properties.

Densities of the pure components and binary mixture were measured at atmospheric pressure with Anton Paar DSA-4500MA digital vibrating U-tube densimeter. The densitometer has a well-defined thermoelectric temperature control system. The uncertainty in the density measurement is $\pm 0.0001 \mathrm{~g} \cdot \mathrm{cm}^{-3}$. The apparatus was calibrated by measuring the density of Millipore quality water and ambient air. The surface tension of the pure components and binary mixtures was measured with a tensiometer by plate type method (Hanging drop tensiometer method, kruss k9, Germany) with a precision of $0.01 \mathrm{mN} / \mathrm{m}$. The apparatus was calibrated by measuring the surface tension of Millipore quality water at ambient temperature. Refractive index of the pure components and binary mixtures was determined at ambient temperature using an automatic refractometer AD-13 model (ABBEMAT-WR Dr. Kernchen), with an uncertainty of \pm 0.00004 .

Samples were prepared by transferring known mass of the pure liquids into stoppered bottles via syringe. The stoppered bottles are closed with screw caps to seal and prevent evaporation. All weighing was carried out in a balance (Mettler Toledo AT 261) with an accuracy of $\pm 10^{-4} \mathrm{gm}$. Previous experiments [18] showed that equilibrium was attained after 6 hours of stirring at $100 \mathrm{rpm}$ at temperature at $298.15 \mathrm{~K}$, using circulating water bath along with automatic controller. Equilibrium was attained by keeping the mixture settled for 12 hours during which good contact was obtained between two pure components. Samples from the homogeneous mixture were withdrawn using syringes for the thermodynamic phase behavior studies. All the samples were prepared immediately prior to performing density, surface tension, and refractive index measurements so as to prevent variation in composition due to water/air retention via the hygroscopic IL.

\section{Computational Details}

The sigma profile generation and methodologies are given in detail in our previously published work on ionic liquid systems [12-15]. The SCDs obtained in COSMO calculations are given for each segment. Thereafter the expression for chemical potential of segment in the mixture phase is derived in which interaction energies between segments are calculated from COSMO-RS. The chemical potential of each molecule is then obtained by summing the contributions of the segments. 
TABLE 1: Name and structure of the studied ionic liquid.

\begin{tabular}{|c|c|c|c|c|c|c|c|c|}
\hline S. no. & Name & Structure & Acronym & Mole. for & $\begin{array}{l}\text { Mol.wt } \\
\text { (g/mole) }\end{array}$ & CASRN $^{a}$ & $\begin{array}{l}\text { Density } \\
\left(\mathrm{g} / \mathrm{cm}^{3}\right)\end{array}$ & Appearance \\
\hline 01 & $\begin{array}{l}\text { 1-Ethyl-3- } \\
\text { methylimidazolium } \\
\text { thiocyanate }\end{array}$ & & [EMIM] [SCN] & $\mathrm{C}_{7} \mathrm{H}_{11} \mathrm{~N}_{3} \mathrm{~S}$ & 169.25 & $143314-17-4$ & 1.027 & Liquid \\
\hline
\end{tabular}

TABLE 2: Comparison of measured density $\rho$ as a function of temperature with literature data for pure compounds.

\begin{tabular}{|c|c|c|c|c|c|c|}
\hline & $\begin{array}{c}T(\mathrm{~K}) \\
298.15 \\
\rho\left(\mathrm{g} \mathrm{cm}^{-3}\right)\end{array}$ & 303.15 & 308.15 & 313.15 & 318.15 & 323.15 \\
\hline \multicolumn{7}{|c|}{ 1-ethyl-3-methylimidazolium thiocyanate [EMIM] [SCN] } \\
\hline Measured & 1.1345 & 1.1313 & 1.1279 & 1.1246 & 1.1213 & 1.1179 \\
\hline Reported $^{\mathrm{a}}$ & 1.1168 & NA & 1.1107 & NA & 1.1047 & NA \\
\hline \multicolumn{7}{|c|}{ Pyridine (PY) } \\
\hline Measured & 0.9783 & 0.9732 & 0.9682 & 0.9631 & 0.9581 & 0.9581 \\
\hline Reported & $0.9780^{\mathrm{b}}$ & NA & NA & NA & NA & NA \\
\hline \multicolumn{7}{|c|}{ Pyrrole (PYRR) } \\
\hline Measured & 0.9655 & 0.9611 & 0.9568 & 0.9524 & 0.9479 & 0.9436 \\
\hline Reported & $0.9733^{\mathrm{b}}$ & NA & NA & NA & NA & NA \\
\hline \multicolumn{7}{|c|}{ Quinoline (Qu) } \\
\hline Measured & 1.0883 & 1.0843 & 1.0804 & 1.0764 & 1.0724 & 1.0685 \\
\hline Reported & $1.0929^{\mathrm{b}}$ & NA & NA & NA & NA & NA \\
\hline \multicolumn{7}{|c|}{ Indoline (INDO) } \\
\hline Measured & 1.0883 & 1.0843 & 1.0804 & 1.0764 & 1.0724 & 1.0685 \\
\hline Reported & NA & NA & NA & NA & NA & NA \\
\hline \multicolumn{7}{|c|}{ Thiophene (TS) } \\
\hline Measured & 1.0584 & 1.0525 & 1.0465 & 1.0405 & 1.0344 & 1.0284 \\
\hline Reported & $1.0585^{\mathrm{c}}$ & NA & NA & NA & NA & NA \\
\hline \multicolumn{7}{|c|}{ Water } \\
\hline Measured & 0.9971 & 0.9957 & 0.9939 & 0.9918 & 0.9879 & 0.9849 \\
\hline Reported & $0.9971^{\mathrm{d}}$ & NA & NA & NA & NA & NA \\
\hline
\end{tabular}

${ }^{\mathrm{a}}[15] ;{ }^{\mathrm{b}}[16] ;{ }^{\mathrm{c}}[17] ;{ }^{\mathrm{d}}[18] ;$ NA. not found.

For the prediction, a complete dissociation of ionic liquids into cations and anions was assumed as in our earlier work [13-15].The activity coefficient of any component in the mixture is then given by

$$
\ln \gamma_{i / S}=n_{i} \sum_{\sigma} p_{i}(\sigma)\left[\ln \Gamma_{S}(\sigma)-\Gamma_{i}(\sigma)\right]+\ln \gamma_{i / S}^{\mathrm{SG}}
$$

Here $\ln \Gamma_{S}(\sigma)$ and $\ln \Gamma_{i}(\sigma)$ refer to the activity coefficient of the segment in the mixture and compound $i$, respectively. $p_{i}(\sigma)$ refers to the sigma profile of the component $i$, and $n_{i}$ implies the contribution of number of segments for component $i$. Thereafter the Staverman-Guggenheim term for the combinatorial contribution $\left[\ln \gamma_{i / S}^{S G}\right]$ is calculated using the following equation:

$$
\ln \gamma_{i / S}^{\mathrm{SG}}=\ln \frac{\phi_{i}}{x_{i}}+\frac{z}{2} q_{i} \ln \frac{\theta_{i}}{\phi_{i}}+l_{i}-\frac{\phi_{i}}{x_{i}} \sum_{j} x_{j} l_{j}
$$

where

$\theta_{i}=\frac{x_{i} q_{i}}{\sum_{j} x_{j} q_{j}}, \quad \phi_{i}=\frac{x_{i} r_{i}}{\sum_{j} x_{j} r_{j}}, \quad l_{i}=\frac{z}{2}\left(\left(r_{i}-q_{i}\right)-\left(r_{i}-1\right)\right)$.

Here $r_{i}$ and $q_{i}$ are normalized using the volume $\left(66.69 \mathrm{~A}^{2}\right)$ and surface area $\left(79.53 \mathrm{~A}^{2}\right)$ of a functional group, that is, $r_{i}=$ COSMO Volume/66.69, $q_{i}=$ COSMO Area/79.53. The COSMO volume and COSMO area are taken from the output of the COSMO file. COSMO-RS thereafter estimates the activity coefficients of the components in the liquid-liquid system according to (2).

\section{Results and Discussion}

4.1. Pure Compounds and Their Binary Mixtures. Experimental densities of pure [EMIM][SCN], aromatic nitrogen, aromatic sulphur species, and water have been measured 
TABle 3: Surface tension $\sigma$ and refractive index $n D$ for pure compounds of the binary system studied in this work at $298.15 \mathrm{~K}$ and atmospheric pressure.

\begin{tabular}{lcccc}
\hline & \multicolumn{2}{c}{$\sigma\left(\mathrm{mN} \mathrm{m}^{-1}\right)$} & \multicolumn{2}{c}{$n D$} \\
& $\begin{array}{c}\text { Present } \\
\text { study }\end{array}$ & $\begin{array}{c}\text { Literature } \\
\text { value }\end{array}$ & $\begin{array}{c}\text { Present } \\
\text { study }\end{array}$ & $\begin{array}{c}\text { Literature } \\
\text { value }\end{array}$ \\
\hline [EMIM][SCN] & 53.1 & $57.76^{*}$ & 1.5377 & NA \\
Pyridine (PY) & 37.5 & NA & 1.5013 & NA \\
Pyrrole (PYRR) & 41.7 & NA & 1.4884 & NA \\
Quinoline (QU) & 46.5 & NA & 1.4997 & NA \\
Indoline (INDO) & 32.6 & NA & 1.5867 & NA \\
Thiophene (TS) & 31.7 & NA & 1.5271 & $1.5255^{\mathrm{e}, \mathrm{f}}$ \\
Water & 72.1 & $71.1^{\mathrm{g}}$ & 1.3357 & $\mathrm{NA}$ \\
\hline
\end{tabular}

${ }^{*}[15] ;{ }^{\mathrm{e}}[16] ;{ }^{\mathrm{f}}[17] ;{ }^{\mathrm{g}}[19] ;$ NA. not found.

and benchmarked at different temperatures (Tables 2 and 3 ). Besides the effect of temperature and experimental method, the presence of trace amount of impurities such as water or ions provides a remarkable effect on thermodynamic properties [20]. Table 2 shows the comparison of measured densities for pure [EMIM] [SCN] with temperature. Table 3 shows the comparison between experimental and literature data of the pure aromatic nitrogen/sulphur and water at $298.15 \mathrm{~K}$. This agrees will with data reported for pyridine, pyrrole, and quinoline [21]. This also matches with the experimental data obtained earlier for thiophene [22] and water [23]. It was observed that the density $\rho$ of pure ionic liquid is higher than that of water and also than that of aromatic nitrogen/sulphur compounds. Table 3 shows a comparison for the surface tension $\sigma$ and refractive index $n D$ of pure components with literature data at $298.15 \mathrm{~K}$. The surface tension and refractive index were compared successfully against the known value of water [21] and thiophene [22], respectively. The difference between experimental and literature data for pure [EMIM] [SCN] can be explained by the thermodynamic properties which strongly depend on water and other impurities.

Experimental densities over the entire composition range for [EMIM] [SCN] (1) + PY(2) (Table 4(a)), [EMIM] [SCN](1)+ $\operatorname{PYRR}(2)($ Table 4(b)), [EMIM] [SCN] (1)+QU(2) (Table 4(c)), [EMIM] [SCN] (1)+INDO(2) (Table 4(d)), [EMIM] [SCN] (1)+ TS(2) (Table 4(e)), and [EMIM] [SCN] (1) + water(2) (Table 4(f)) have been determined at temperature of $T=(298.15$ to 323.15$) \mathrm{K}$. The surface tension $\sigma$ and refractive index $n D$ of the pure component and its mixtures were determined at $298.15 \mathrm{~K}$ from 0.2 to 0.8 mole fraction of [EMIM] [SCN] (1). Additionally we have studied the binary mixtures of $[\mathrm{EMIM}][\mathrm{SCN}](1)+$ water(2) which is more helpful in the purification and regeneration of [EMIM] [SCN] [22].

4.2. Thermal Expansion " $\alpha$ ". The coefficient of thermal expansion of the [EMIM] [SCN] $\alpha$ is shown in Figure 1. The densities of pure [EMIM] [SCN] decrease linearly $\left(R^{2}=1\right)$

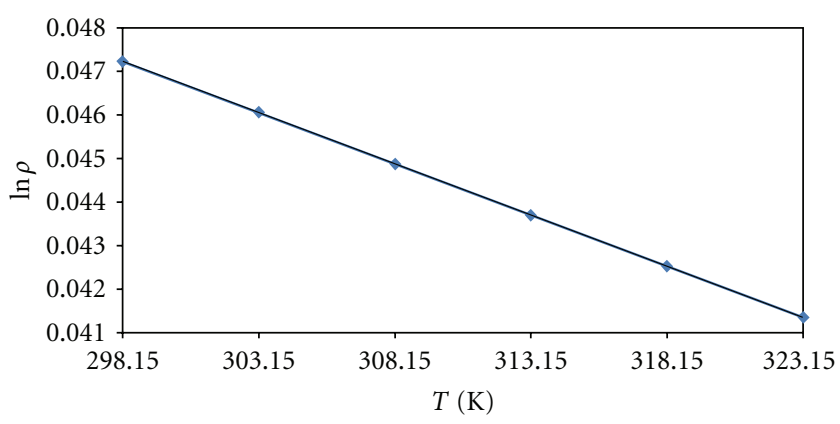

FIGURE 1: Experimental values of $\ln \rho$ of pure [EMIM] [SCN] ionic liquid versus temperature of (298.15 to 323.15$) \mathrm{K}$.

with increasing temperature. The value of $\alpha=0.117 \mathrm{~K}^{-1}$ (ionic liquid) was calculated from

$$
\alpha=\frac{1}{V}\left(\frac{\partial V}{\partial T}\right)_{P}=-\frac{1}{\rho}\left(\frac{\partial \rho}{\partial T}\right)_{P}
$$

where $V$ is the molar volume of the pure fluid, $\rho$ is the density of the pure fluid, and subscript $P$ indicates constant pressure. The volume expansivity of [EMIM] [SCN] was calculated using the measured density observed at different temperatures. The volume expansivity of [EMIM] $[\mathrm{SCN}]$ was found to be independent of temperature (Figure 1). These observations are more consistent with the studies of Rodríguez and Brennecke [24], Wandschneider et al. [25], and Pereiro et al. [26, 27].

4.3. Effect of Composition on Density and Excess Molar Volume. Table 2 shows the experimental densities of pure [EMIM][SCN], aromatic nitrogen, aromatic sulphur, and water at temperatures of $T=(298.15$ to 323.15$) \mathrm{K}$. It can be seen that the density of all the pure components including ionic liquid decreases linearly with increasing temperature. Tables 4(a) to 4(f) show the experimental densities for six binary systems studied as a function of temperature over the studied mole fraction (0.2 to 0.8) of [EMIM] [SCN]. For all the compounds with ILs, the densities varied linearly in the entire mole fraction with increasing temperature. This behavior can be explained by the efficient structural interaction of [EMIM] [SCN] with aromatic structure of nitrogen/sulphur and water via packing effects [13-16, 18, $19,28,29$ ] (Scheme 1). Additionally it can be a result of smaller size of cation [18] and anion providing better interaction with aromatic compounds. A small size of IL molecule has a significant influence on the changing densities of the mixture with an increase in temperature over the studied mole fraction (0.2 to 0.8$)$ of IL. Besides the hydrogen bonding strongly depends on the temperature [30] as compared to $\mathrm{CH}-\pi$ bond interaction $[12-14,31-33]$ and $\pi-\pi$ bond interactions $[13,14,27-29]$. These similar trends were observed for other binary systems [24, 28, 29].

The measured densities of binary mixtures over the studied mole fraction (0.2 to 0.8$)$ of [EMIM] [SCN] at several temperature are presented in Tables 4(a) to 4(f). The binary mixture of $[\mathrm{EMIM}][\mathrm{SCN}](1)+$ pyrrole(2) system shows 
TABLE 4

(a) Experimental density $\rho$ and excess molar volume $V_{m}^{E}$, for the binary system [EMIM] [SCN](1) + Pyridine (2).

\begin{tabular}{|c|c|c|c|c|c|c|c|c|c|}
\hline$x_{\mathrm{IL}}$ & $\begin{array}{c}\rho \\
\mathrm{g} \mathrm{cm}^{-3} \\
\end{array}$ & $\begin{array}{c}V_{m}^{E} \\
\mathrm{~cm}^{3} \mathrm{~mol}^{-1} \\
\end{array}$ & $\gamma_{\text {COSMO-RS }}$ & $\begin{array}{c}\rho \\
\mathrm{g} \mathrm{cm}^{-3}\end{array}$ & $\begin{array}{c}V_{m}^{E} \\
\mathrm{~cm}^{3} \mathrm{~mol}^{-1} \\
\end{array}$ & $\gamma_{\text {COSMO-RS }}$ & $\begin{array}{c}\rho \\
\mathrm{g} \mathrm{cm}^{-3} \\
\end{array}$ & $\begin{array}{c}V_{m}^{E} \\
\mathrm{~cm}^{3} \mathrm{~mol}^{-1} \\
\end{array}$ & $\gamma_{\text {COSMO-RS }}$ \\
\hline & \multicolumn{3}{|c|}{$T=298.15 \mathrm{~K}$} & \multicolumn{3}{|c|}{$T=303.15 \mathrm{~K}$} & \multicolumn{3}{|c|}{$T=308.15 \mathrm{~K}$} \\
\hline 0.2 & 1.0452 & -0.0434 & 1.1988 & 1.0412 & -0.0396 & 1.2007 & 1.0372 & -0.0357 & 1.2025 \\
\hline 0.3 & 1.0601 & -0.0578 & 1.3841 & 1.0563 & -0.0541 & 1.3874 & 1.0525 & -0.0504 & 1.3900 \\
\hline 0.4 & 1.0726 & -0.0699 & 1.5733 & 1.0691 & -0.0664 & 1.5788 & 1.0655 & -0.0630 & 1.5833 \\
\hline 0.5 & 1.0823 & -0.0795 & 1.7402 & 1.0788 & -0.0762 & 1.7493 & 1.0754 & -0.0728 & 1.7570 \\
\hline 0.6 & 1.0928 & -0.0900 & 1.8689 & 1.0895 & -0.0868 & 1.8822 & 1.0862 & -0.0836 & 1.8950 \\
\hline 0.7 & 1.0965 & -0.0945 & 1.9504 & 1.0933 & -0.0913 & 1.9694 & 1.0900 & -0.0881 & 1.9879 \\
\hline \multirow[t]{2}{*}{0.8} & 1.1021 & -0.1007 & 1.9863 & 1.0989 & -0.0975 & 2.0117 & 1.0957 & -0.0944 & 2.0362 \\
\hline & \multicolumn{3}{|c|}{$T=313.15 \mathrm{~K}$} & \multicolumn{3}{|c|}{$T=318.15 \mathrm{~K}$} & \multicolumn{3}{|c|}{$T=323.15 \mathrm{~K}$} \\
\hline 0.2 & 1.0332 & -0.0319 & 1.2039 & 1.0293 & -0.0281 & 1.2053 & 1.0253 & -0.0242 & 1.2066 \\
\hline 0.3 & 1.0488 & -0.0468 & 1.3923 & 1.0450 & -0.0431 & 1.3946 & 1.0412 & -0.0395 & 1.3965 \\
\hline 0.4 & 1.0620 & -0.0596 & 1.5877 & 1.0585 & -0.0562 & 1.5911 & 1.0550 & -0.0528 & 1.5944 \\
\hline 0.5 & 1.0720 & -0.0695 & 1.7645 & 1.0686 & -0.0662 & 1.7706 & 1.0653 & -0.0629 & 1.7765 \\
\hline 0.6 & 1.0830 & -0.0803 & 1.9061 & 1.0797 & -0.0771 & 1.9169 & 1.0765 & -0.0739 & 1.9259 \\
\hline 0.7 & 1.0868 & -0.0849 & 2.0042 & 1.0836 & -0.0817 & 2.0201 & 1.0804 & -0.0785 & 2.0339 \\
\hline 0.8 & 1.0925 & -0.0912 & 2.0591 & 1.0894 & -0.0881 & 2.0799 & 1.0862 & -0.0850 & 2.1000 \\
\hline
\end{tabular}

(b) Experimental density $\rho$ and excess molar volume $V_{m}^{E}$, for the binary system [EMIM] [SCN](1) + Pyrrole (2).

\begin{tabular}{|c|c|c|c|c|c|c|c|c|c|}
\hline$x_{\mathrm{IL}}$ & $\begin{array}{c}\rho \\
\mathrm{g} \mathrm{cm}^{-3}\end{array}$ & $\begin{array}{c}V_{m}^{E} \\
\mathrm{~cm}^{3} \mathrm{~mol}^{-1} \\
\end{array}$ & $\gamma_{\text {COSMO-RS }}$ & $\begin{array}{c}\rho \\
\mathrm{g} \mathrm{cm}^{-3} \\
\end{array}$ & $\begin{array}{c}V_{m}^{E} \\
\mathrm{~cm}^{3} \mathrm{~mol}^{-1} \\
\end{array}$ & $\gamma_{\text {COSMO-RS }}$ & $\begin{array}{c}\rho \\
\mathrm{g} \mathrm{cm}^{-3}\end{array}$ & $\begin{array}{c}V_{m}^{E} \\
\mathrm{~cm}^{3} \mathrm{~mol}^{-1}\end{array}$ & $\gamma_{\text {COSMO-RS }}$ \\
\hline & \multicolumn{3}{|c|}{$T=298.15 \mathrm{~K}$} & \multicolumn{3}{|c|}{$T=303.15 \mathrm{~K}$} & \multicolumn{3}{|c|}{$T=308.15 \mathrm{~K}$} \\
\hline 0.2 & 1.0284 & -0.0275 & 0.3445 & 1.0247 & -0.0239 & 0.3552 & 1.0210 & -0.0203 & 0.3659 \\
\hline 0.3 & 1.0492 & -0.0472 & 0.2176 & 1.0457 & -0.0440 & 0.2271 & 1.0422 & -0.0406 & 0.2367 \\
\hline 0.4 & 1.0562 & -0.0543 & 0.1522 & 1.0528 & -0.0512 & 0.1604 & 1.0493 & -0.0478 & 0.1687 \\
\hline 0.5 & 1.0736 & -0.0708 & 0.1124 & 1.0703 & -0.0680 & 0.1194 & 1.0669 & -0.0648 & 0.1266 \\
\hline 0.6 & 1.0886 & -0.0853 & 0.0858 & 1.0854 & -0.0827 & 0.0919 & 1.0822 & -0.0796 & 0.0981 \\
\hline 0.7 & 1.0945 & -0.0918 & 0.0671 & 1.0913 & -0.0893 & 0.0723 & 1.0882 & -0.0861 & 0.0777 \\
\hline \multirow[t]{2}{*}{0.8} & 1.1035 & -0.1018 & 0.0535 & 1.1003 & -0.0986 & 0.058 & 1.0972 & -0.0955 & 0.0626 \\
\hline & \multicolumn{3}{|c|}{$T=313.15 \mathrm{~K}$} & \multicolumn{3}{|c|}{$T=318.15 \mathrm{~K}$} & \multicolumn{3}{|c|}{$T=323.15 \mathrm{~K}$} \\
\hline 0.2 & 1.0174 & -0.0168 & 0.3764 & 1.0137 & -0.0133 & 0.3869 & 1.0101 & -0.0097 & 0.3973 \\
\hline 0.3 & 1.0388 & -0.0373 & 0.2464 & 1.0353 & -0.0339 & 0.256 & 1.0319 & -0.0306 & 0.2657 \\
\hline 0.4 & 1.0458 & -0.0444 & 0.1771 & 1.0424 & -0.0411 & 0.1857 & 1.0390 & -0.0377 & 0.1942 \\
\hline 0.5 & 1.0636 & -0.0616 & 0.134 & 1.0604 & -0.0584 & 0.1415 & 1.0571 & -0.0552 & 0.1491 \\
\hline 0.6 & 1.0790 & -0.0764 & 0.1045 & 1.0758 & -0.0733 & 0.111 & 1.0726 & -0.0702 & 0.1177 \\
\hline 0.7 & 1.0850 & -0.0830 & 0.0832 & 1.0819 & -0.0800 & 0.089 & 1.0788 & -0.0769 & 0.0948 \\
\hline 0.8 & 1.0941 & -0.0925 & 0.0675 & 1.0910 & -0.0894 & 0.0725 & 1.0879 & -0.0864 & 0.0777 \\
\hline
\end{tabular}


(c) Experimental density $\rho$ and excess molar volume $V_{m}^{E}$ for the binary system [EMIM] [SCN](1) + Quinoline (2).

\begin{tabular}{|c|c|c|c|c|c|c|c|c|c|}
\hline$x_{\mathrm{IL}}$ & $\begin{array}{c}\rho \\
\mathrm{gcm}^{-3}\end{array}$ & $\begin{array}{c}V_{m}^{E} \\
\mathrm{~cm}^{3} \mathrm{~mol}^{-1} \\
\end{array}$ & $\gamma_{\text {COSMO-RS }}$ & $\begin{array}{c}\rho \\
\mathrm{g} \mathrm{cm}^{-3}\end{array}$ & $\begin{array}{c}V_{m}^{E} \\
\mathrm{~cm}^{3} \mathrm{~mol}^{-1}\end{array}$ & $\gamma_{\text {COSMO-RS }}$ & $\begin{array}{c}\rho \\
\mathrm{g} \mathrm{cm}^{-3}\end{array}$ & $\begin{array}{c}V_{m}^{E} \\
\mathrm{~cm}^{3} \mathrm{~mol}^{-1}\end{array}$ & $\gamma_{\text {COSMO-RS }}$ \\
\hline & \multicolumn{3}{|c|}{$T=298.15 \mathrm{~K}$} & \multicolumn{3}{|c|}{$T=303.15 \mathrm{~K}$} & \multicolumn{3}{|c|}{$T=308.15 \mathrm{~K}$} \\
\hline 0.2 & 1.1084 & -0.1070 & 1.4345 & 1.1049 & -0.1034 & 1.4326 & 1.1012 & -0.0998 & 1.4307 \\
\hline 0.3 & 1.1122 & -0.1106 & 1.9141 & 1.1087 & -0.1071 & 1.9102 & 1.1053 & -0.1037 & 1.9062 \\
\hline 0.4 & 1.1140 & -0.1125 & 2.5577 & 1.1106 & -0.1090 & 2.5528 & 1.1071 & -0.1056 & 2.5477 \\
\hline 0.5 & 1.1163 & -0.1148 & 3.3339 & 1.1131 & -0.1116 & 3.3324 & 1.1098 & -0.1083 & 3.3304 \\
\hline 0.6 & 1.1170 & -0.1157 & 4.1652 & 1.1138 & -0.1125 & 4.1776 & 1.1106 & -0.1093 & 4.1854 \\
\hline 0.7 & 1.1172 & -0.1161 & 4.9321 & 1.1141 & -0.1130 & 4.9702 & 1.1109 & -0.1098 & 5.0011 \\
\hline \multirow[t]{2}{*}{0.8} & 1.1173 & -0.1165 & 5.5048 & 1.1141 & -0.1133 & 5.5815 & 1.1110 & -0.1102 & 5.6486 \\
\hline & \multicolumn{3}{|c|}{$T=313.15 \mathrm{~K}$} & \multicolumn{3}{|c|}{$T=318.15 \mathrm{~K}$} & \multicolumn{3}{|c|}{$T=323.15 \mathrm{~K}$} \\
\hline 0.2 & 1.0976 & -0.0963 & 1.4285 & 1.0940 & -0.0927 & 1.4264 & 1.0904 & -0.0891 & 1.424 \\
\hline 0.3 & 1.1018 & -0.1003 & 1.9016 & 1.0983 & -0.0968 & 1.897 & 1.0948 & -0.0934 & 1.8918 \\
\hline 0.4 & 1.1037 & -0.1022 & 2.5414 & 1.1002 & -0.0988 & 2.535 & 1.0968 & -0.0954 & 2.5274 \\
\hline 0.5 & 1.1065 & -0.1050 & 3.3258 & 1.1033 & -0.1018 & 3.321 & 1.1000 & -0.0986 & 3.3139 \\
\hline 0.6 & 1.1074 & -0.1061 & 4.1923 & 1.1042 & -0.1029 & 4.1949 & 1.1010 & -0.0997 & 4.1969 \\
\hline 0.7 & 1.1077 & -0.1067 & 5.0304 & 1.1045 & -0.1035 & 5.0529 & 1.1014 & -0.1004 & 5.0742 \\
\hline 0.8 & 1.1079 & -0.1071 & 5.7135 & 1.1047 & -0.1040 & 5.7725 & 1.1016 & -0.1009 & 5.8225 \\
\hline
\end{tabular}

(d) Experimental density $\rho$ and excess molar volume $V_{m}^{E}$ for the binary system [EMIM] [SCN](1) + Indoline (2).

\begin{tabular}{|c|c|c|c|c|c|c|c|c|c|}
\hline$x_{\mathrm{IL}}$ & $\begin{array}{c}\rho \\
\mathrm{g} \mathrm{cm}^{-3}\end{array}$ & $\begin{array}{c}V_{m}^{E} \\
\mathrm{~cm}^{3} \mathrm{~mol}^{-1}\end{array}$ & $\gamma_{\text {COSMO-RS }}$ & $\begin{array}{c}\rho \\
\mathrm{g} \mathrm{cm}^{-3}\end{array}$ & $\begin{array}{c}V_{m}^{E} \\
\mathrm{~cm}^{3} \mathrm{~mol}^{-1}\end{array}$ & $\gamma_{\text {COSMO-RS }}$ & $\begin{array}{c}\rho \\
\mathrm{g} \mathrm{cm}^{-3}\end{array}$ & $\begin{array}{c}V_{m}^{E} \\
\mathrm{~cm}^{3} \mathrm{~mol}^{-1} \\
\end{array}$ & $\gamma_{\text {COSMO-RS }}$ \\
\hline & \multicolumn{3}{|c|}{$T=298.15 \mathrm{~K}$} & \multicolumn{3}{|c|}{$T=303.15 \mathrm{~K}$} & \multicolumn{3}{|c|}{$T=308.15 \mathrm{~K}$} \\
\hline 0.2 & 1.0838 & -0.0846 & 1.2640 & 1.0802 & -0.0809 & 1.2659 & 1.0765 & -0.0772 & 1.2675 \\
\hline 0.3 & 1.0927 & -0.0930 & 1.5451 & 1.0893 & -0.0896 & 1.5484 & 1.0858 & -0.0861 & 1.5511 \\
\hline 0.4 & 1.0992 & -0.0991 & 1.8866 & 1.0959 & -0.0959 & 1.8931 & 1.0926 & -0.0925 & 1.8987 \\
\hline 0.5 & 1.1041 & -0.1039 & 2.2431 & 1.1009 & -0.1006 & 2.2568 & 1.0976 & -0.0973 & 2.2687 \\
\hline 0.6 & 1.1061 & -0.1059 & 2.5539 & 1.1029 & -0.1027 & 2.5800 & 1.0997 & -0.0995 & 2.6033 \\
\hline 0.7 & 1.1093 & -0.1090 & 2.7565 & 1.1061 & -0.1059 & 2.8004 & 1.1030 & -0.1027 & 2.8406 \\
\hline \multirow[t]{2}{*}{0.8} & 1.1131 & -0.1127 & 2.8091 & 1.1100 & -0.1096 & 2.8725 & 1.1069 & -0.1065 & 2.9346 \\
\hline & \multicolumn{3}{|c|}{$T=313.15 \mathrm{~K}$} & \multicolumn{3}{|c|}{$T=318.15 \mathrm{~K}$} & \multicolumn{3}{|c|}{$T=323.15 \mathrm{~K}$} \\
\hline 0.2 & 1.0729 & -0.0735 & 1.2690 & 1.0693 & -0.0699 & 1.2702 & 1.0656 & -0.0662 & 1.2712 \\
\hline 0.3 & 1.0824 & -0.0826 & 1.5536 & 1.0789 & -0.0791 & 1.5555 & 1.0754 & -0.0755 & 1.5572 \\
\hline 0.4 & 1.0892 & -0.0891 & 1.9038 & 1.0859 & -0.0858 & 1.9080 & 1.0826 & -0.0825 & 1.9117 \\
\hline 0.5 & 1.0944 & -0.0941 & 2.2799 & 1.0911 & -0.0908 & 2.2895 & 1.0879 & -0.0876 & 2.2984 \\
\hline 0.6 & 1.0965 & -0.0962 & 2.6257 & 1.0933 & -0.0931 & 2.6455 & 1.0901 & -0.0899 & 2.6644 \\
\hline 0.7 & 1.0999 & -0.0996 & 2.8796 & 1.0967 & -0.0965 & 2.9150 & 1.0936 & -0.0934 & 2.9493 \\
\hline 0.8 & 1.1038 & -0.1034 & 2.9926 & 1.1008 & -0.1004 & 3.0492 & 1.0977 & -0.0974 & 3.1032 \\
\hline
\end{tabular}


(e) Experimental density $\rho$ and excess molar volume $V_{m}^{E}$, for the binary system [EMIM] [SCN](1) + Thiophene (2).

\begin{tabular}{|c|c|c|c|c|c|c|c|c|c|}
\hline$x_{\mathrm{IL}}$ & $\begin{array}{c}\rho \\
\mathrm{g} \mathrm{cm}^{-3} \\
\end{array}$ & $\begin{array}{c}V_{m}^{E} \\
\mathrm{~cm}^{3} \mathrm{~mol}^{-1} \\
\end{array}$ & $\gamma_{\text {COSMO-RS }}$ & $\begin{array}{c}\rho \\
\mathrm{g} \mathrm{cm}^{-3}\end{array}$ & $\begin{array}{c}V_{m}^{E} \\
\mathrm{~cm}^{3} \mathrm{~mol}^{-1} \\
\end{array}$ & $\gamma_{\mathrm{COSMO}-\mathrm{RS}}$ & $\begin{array}{c}\rho \\
\mathrm{g} \mathrm{cm}^{-3} \\
\end{array}$ & $\begin{array}{c}V_{m}^{E} \\
\mathrm{~cm}^{3} \mathrm{~mol}^{-1} \\
\end{array}$ & $\gamma_{\text {COSMO-RS }}$ \\
\hline & \multicolumn{3}{|c|}{$T=298.15 \mathrm{~K}$} & \multicolumn{3}{|c|}{$T=303.15 \mathrm{~K}$} & \multicolumn{3}{|c|}{$T=308.15 \mathrm{~K}$} \\
\hline 0.2 & 1.1107 & -0.1066 & 1.6572 & 1.1067 & -0.1026 & 1.6502 & 1.1023 & -0.0983 & 1.6432 \\
\hline 0.3 & 1.1061 & -0.1031 & 2.0822 & 1.1024 & -0.0994 & 2.0738 & 1.0986 & -0.0956 & 2.0654 \\
\hline 0.4 & 1.1122 & -0.1090 & 2.4632 & 1.1085 & -0.1054 & 2.4581 & 1.1049 & -0.1017 & 2.4518 \\
\hline 0.5 & 1.1144 & -0.1116 & 2.7427 & 1.1107 & -0.1078 & 2.7449 & 1.1069 & -0.1040 & 2.7464 \\
\hline 0.6 & 1.1150 & -0.1127 & 2.8916 & 1.1119 & -0.1095 & 2.9055 & 1.1087 & -0.1062 & 2.9184 \\
\hline 0.7 & 1.1157 & -0.1139 & 2.9142 & 1.1124 & -0.1105 & 2.9428 & 1.1090 & -0.1071 & 2.9688 \\
\hline \multirow[t]{2}{*}{0.8} & 1.1166 & -0.1152 & 2.8414 & 1.1133 & -0.1120 & 2.8824 & 1.1101 & -0.1087 & 2.9211 \\
\hline & \multicolumn{3}{|c|}{$T=313.15 \mathrm{~K}$} & \multicolumn{3}{|c|}{$T=318.15 \mathrm{~K}$} & \multicolumn{3}{|c|}{$T=323.15 \mathrm{~K}$} \\
\hline 0.2 & 1.0980 & -0.0940 & 1.6362 & 1.0935 & -0.0896 & 1.6292 & 1.0889 & -0.0851 & 1.6222 \\
\hline 0.3 & 1.0947 & -0.0917 & 2.0566 & 1.0909 & -0.0879 & 2.0477 & 1.0870 & -0.0840 & 2.0384 \\
\hline 0.4 & 1.1012 & -0.0981 & 2.4452 & 1.0976 & -0.0944 & 2.4376 & 1.0940 & -0.0908 & 2.4298 \\
\hline 0.5 & 1.1031 & -0.1003 & 2.7459 & 1.0993 & -0.0965 & 2.7449 & 1.0955 & -0.0928 & 2.7421 \\
\hline 0.6 & 1.1055 & -0.1030 & 2.9285 & 1.1023 & -0.0998 & 2.9376 & 1.0992 & -0.0967 & 2.945 \\
\hline 0.7 & 1.1056 & -0.1038 & 2.9915 & 1.1023 & -0.1004 & 3.0129 & 1.0990 & -0.0971 & 3.0321 \\
\hline 0.8 & 1.1069 & -0.1055 & 2.9573 & 1.1037 & -0.1023 & 2.9909 & 1.1005 & -0.0991 & 3.0216 \\
\hline
\end{tabular}

(f) Experimental density $\rho$ and excess molar volume $V_{m}^{E}$, for the binary system [EMIM] [SCN](1) + water (2).

\begin{tabular}{|c|c|c|c|c|c|c|c|c|c|}
\hline$x_{\mathrm{IL}}$ & $\begin{array}{c}\rho \\
\mathrm{g} \mathrm{cm}^{-3} \\
\end{array}$ & $\begin{array}{c}V_{m}^{E} \\
\mathrm{~cm}^{3} \mathrm{~mol}^{-1} \\
\end{array}$ & $\gamma_{\text {COSMO-RS }}$ & $\begin{array}{c}\rho \\
\mathrm{g} \mathrm{cm}^{-3}\end{array}$ & $\begin{array}{c}V_{m}^{E} \\
\mathrm{~cm}^{3} \mathrm{~mol}^{-1} \\
\end{array}$ & $\gamma_{\text {COSMO-RS }}$ & $\begin{array}{c}\rho \\
\mathrm{g} \mathrm{cm}^{-3} \\
\end{array}$ & $\begin{array}{c}V_{m}^{E} \\
\mathrm{~cm}^{3} \mathrm{~mol}^{-1} \\
\end{array}$ & $\gamma_{\text {COSMO-RS }}$ \\
\hline & \multicolumn{3}{|c|}{$T=298.15 \mathrm{~K}$} & \multicolumn{3}{|c|}{$T=303.15 \mathrm{~K}$} & \multicolumn{3}{|c|}{$T=308.15 \mathrm{~K}$} \\
\hline 0.2 & 1.0758 & -0.0719 & 0.8053 & 1.0725 & -0.0689 & 0.8106 & 1.0693 & -0.0659 & 0.8157 \\
\hline 0.3 & 1.0893 & -0.0847 & 0.7201 & 1.0861 & -0.0817 & 0.7261 & 1.0828 & -0.0787 & 0.7319 \\
\hline 0.4 & 1.0997 & -0.0947 & 0.6502 & 1.0966 & -0.0918 & 0.6565 & 1.0934 & -0.0888 & 0.6624 \\
\hline 0.5 & 1.1042 & -0.0996 & 0.5916 & 1.1010 & -0.0966 & 0.5978 & 1.0978 & -0.0937 & 0.6038 \\
\hline 0.6 & 1.1066 & -0.1029 & 0.5417 & 1.1035 & -0.0999 & 0.5477 & 1.1004 & -0.0969 & 0.5537 \\
\hline 0.7 & 1.1104 & -0.1074 & 0.4987 & 1.1074 & -0.1044 & 0.5045 & 1.1043 & -0.1015 & 0.5104 \\
\hline \multirow[t]{2}{*}{0.8} & 1.1112 & -0.1092 & 0.4612 & 1.1081 & -0.1062 & 0.4670 & 1.1050 & -0.1032 & 0.4725 \\
\hline & \multicolumn{3}{|c|}{$T=313.15 \mathrm{~K}$} & \multicolumn{3}{|c|}{$T=318.15 \mathrm{~K}$} & \multicolumn{3}{|c|}{$T=323.15 \mathrm{~K}$} \\
\hline 0.2 & 1.0659 & -0.0628 & 0.8206 & 1.0626 & -0.0596 & 0.8254 & 1.0591 & -0.0562 & 0.8299 \\
\hline 0.3 & 1.0796 & -0.0756 & 0.7376 & 1.0763 & -0.0725 & 0.7431 & 1.0731 & -0.0694 & 0.7483 \\
\hline 0.4 & 1.0902 & -0.0858 & 0.6683 & 1.0870 & -0.0827 & 0.6740 & 1.0838 & -0.0797 & 0.6794 \\
\hline 0.5 & 1.0946 & -0.0907 & 0.6097 & 1.0915 & -0.0876 & 0.6153 & 1.0883 & -0.0846 & 0.6208 \\
\hline 0.6 & 1.0972 & -0.0939 & 0.5594 & 1.0941 & -0.0909 & 0.5650 & 1.0910 & -0.0879 & 0.5704 \\
\hline 0.7 & 1.1012 & -0.0985 & 0.5159 & 1.0982 & -0.0955 & 0.5214 & 1.0951 & -0.0925 & 0.5266 \\
\hline 0.8 & 1.1020 & -0.1002 & 0.4779 & 1.0989 & -0.0972 & 0.4833 & 1.0958 & -0.0942 & 0.4884 \\
\hline
\end{tabular}




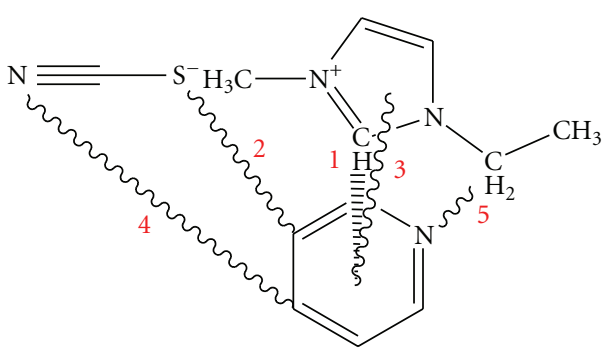

Six membered ring

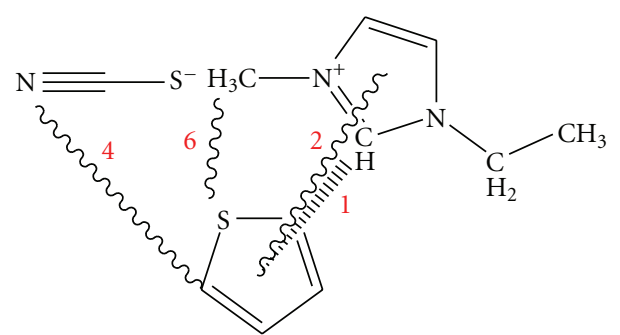

Five membered ring

Possible weak interactions are:

(1) $\mathrm{CH}$ (cation) $----\pi$ (pyridine)

(2) $\mathrm{S}$ (anion) $----\mathrm{H}$ (pyridine)

(3) $\pi$ (cation) $---\pi$ (pyridine)

(4) $\mathrm{N}$ (anion) - - - - H (pyridine)

(5) $\mathrm{N}$ (pyridine) - - - - H (cation)

(6) $\mathrm{H}$ (cation) - - - - S (thiophene)

Scheme 1: 1-ethyl-3-methylimidazolium thiocyanate with six membered ring of pyridine and five membered ring of thiophene [9-12, 14, $15]$.

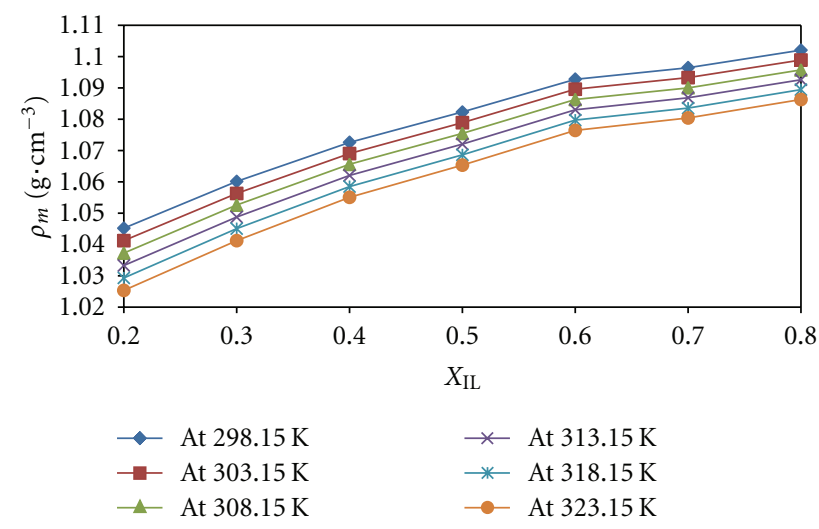

FIGURE 2: Experimental density for the binary system $\{[$ EMIM $][S C N](1)+$ Pyridine(2) $\}$ as a function of mole fraction of the IL at different temperature.

that the composition or mole fraction of [EMIM] [SCN](1) increases with density. This is due to the stronger association between the similar aromatic structure of molecules (i.e., [EMIM] cation and pyrrole molecule). There are many possibilities in [EMIM][SCN] to interact with aromatic or nonaromatic structure compounds such as (1) $\mathrm{CH}-\pi$ interaction [8-10, 26-29], (2) $\pi-\pi$ interaction [9, 10, 29], and (3) $n-\pi$ interaction [8-10], where $n=N, O, F$, and $\mathrm{S}$ atoms are located on the aromatic/nonaromatic compound or ionic liquid structure (Scheme 1). Therefore the measured densities of binary mixture for [EMIM] [SCN](1) + pyridine(2) systems (Figure 2) show a linear trend in the liquid phase at all temperatures, which includes water (Table 4(f)).

Geng et al. [20] had earlier reported that the ratio of van der Waals and electrostatic force plays a significant role in the extraction process. It was found that mixtures of IL with similar or dissimilar structure of molecules for mole fraction 0.3 and above plays a dominating role at temperatures 293.15

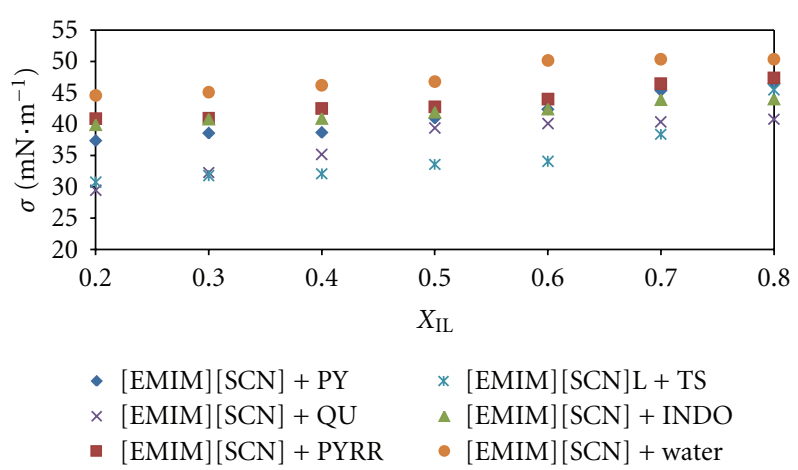

Figure 3: Composition dependence of surface tension of the binary mixture plotted against mole fraction of IL at $298.15 \mathrm{~K}$.

to $318.15 \mathrm{~K}$. On the same lines, the mixture of [EMIM] [SCN] + thiophene shows a similar trend at above 0.3 mole fraction of IL in the mixture.

4.4. Effect of Composition on Surface Tension and Refractive Index. The measured surface tension data for all the studied systems are listed in Table 5 and illustrated in Figure 3. At $x_{\mathrm{IL}}=0.2$ the surface tension of [EMIM][SCN](1) + water system is higher as compared to other systems such as $[$ EMIM $][$ SCN $](1)+$ pyrrole, $[$ EMIM $][S C N](1)+$ pyridine, $[$ EMIM] $[\mathrm{SCN}](1)+$ thiophene, $[\mathrm{EMIM}][\mathrm{SCN}](1)+$ indoline(2), and [EMIM] [SCN](1) + quinoline(2). It should be noted that the strength of van der Waals force is negligible as compared to the sterical hindrance provided by the addition of benzene ring [34-36] in pyrrole/pyridine structure which is accountable upon mixing. Therefore with increasing mole fraction of [EMIM] [SCN], surface tension of mixture increases even if it has dissimilar molecules like indoline (Figure 3 ) and quinoline (Figure 3 ).

It is well-known fact that the strength of $\mathrm{CH}-\pi$ bond and hydrogen bond increases in liquid phase over the entire 
TABLE 5: Experimental surface tension $\sigma$ and deviation of surface tension for the binary systems: (a) [EMIM] [SCN] + Pyridine, (b) $[$ EMIM] $[\mathrm{SCN}]+$ Pyrrole, (c) $[$ EMIM] $[\mathrm{SCN}]+$ Indoline, (d) $[$ EMIM] $[\mathrm{SCN}]+$ Quinoline, (e) $[$ EMIM] $[\mathrm{SCN}]+$ Thiophene, and (f) $[\mathrm{EMIM}][\mathrm{SCN}]+$ Water at $298.15 \mathrm{~K}$.

\begin{tabular}{|c|c|c|c|c|c|c|}
\hline \multirow[t]{2}{*}{$x_{\mathrm{IL}}$} & \multicolumn{2}{|c|}{$[\mathrm{EMIM}][\mathrm{SCN}]+\mathrm{PY}$} & \multicolumn{2}{|c|}{ [EMIM] $[\mathrm{SCN}]+$ PYRR } & \multicolumn{2}{|c|}{$[\mathrm{EMIM}][\mathrm{SCN}]+\mathrm{QU}$} \\
\hline & $\sigma\left(\mathrm{mN} \cdot \mathrm{m}^{-1}\right)$ & $\Delta \sigma\left(\mathrm{mN} \cdot \mathrm{m}^{-1}\right)$ & $\sigma\left(\mathrm{mN} \cdot \mathrm{m}^{-1}\right)$ & $\Delta \sigma\left(\mathrm{mN} \cdot \mathrm{m}^{-1}\right)$ & $\sigma\left(\mathrm{mN} \cdot \mathrm{m}^{-1}\right)$ & $\Delta \sigma\left(\mathrm{mN} \cdot \mathrm{m}^{-1}\right)$ \\
\hline 0.2 & 37.4 & -1.22 & 40.9 & -1.08 & 29.5 & -16.32 \\
\hline 0.3 & 38.6 & -0.58 & 41 & -1.12 & 32.3 & -13.18 \\
\hline 0.4 & 38.7 & -1.04 & 42.5 & 0.24 & 35.2 & -9.94 \\
\hline 0.5 & 41 & 0.7 & 42.8 & 0.4 & 39.4 & -5.4 \\
\hline 0.6 & 42.4 & 1.54 & 44 & 1.46 & 40.1 & -4.36 \\
\hline 0.7 & 45.4 & 3.98 & 46.5 & 3.82 & 40.4 & -3.72 \\
\hline 0.8 & 46.5 & 4.52 & 47.4 & 4.58 & 40.8 & -2.98 \\
\hline \multirow{2}{*}{$x_{\mathrm{IL}}$} & \multicolumn{2}{|c|}{ [EMIM] [SCN] + INDO } & \multicolumn{2}{|c|}{$[\mathrm{EMIM}][\mathrm{SCN}]+\mathrm{TS}$} & \multicolumn{2}{|c|}{ [EMIM][SCN] + Water } \\
\hline & $\sigma\left(\mathrm{mN} \cdot \mathrm{m}^{-1}\right)$ & $\Delta \sigma\left(\mathrm{mN} \cdot \mathrm{m}^{-1}\right)$ & $\sigma\left(\mathrm{mN} \cdot \mathrm{m}^{-1}\right)$ & $\Delta \sigma\left(\mathrm{mN} \cdot \mathrm{m}^{-1}\right)$ & $\sigma\left(\mathrm{mN} \cdot \mathrm{m}^{-1}\right)$ & $\Delta \sigma\left(\mathrm{mN} \cdot \mathrm{m}^{-1}\right)$ \\
\hline 0.2 & 39.9 & 5.2 & 30.8 & -3.18 & 44.6 & -20.1 \\
\hline 0.3 & 40.8 & 5.05 & 31.8 & -3.32 & 45.1 & -16.9 \\
\hline 0.4 & 40.9 & 4.1 & 32.1 & -4.16 & 46.2 & -13.1 \\
\hline 0.5 & 41.9 & 4.05 & 33.6 & -3.8 & 46.8 & -9.8 \\
\hline 0.6 & 42.4 & 3.5 & 34.1 & -4.44 & 50.2 & -3.7 \\
\hline 0.7 & 43.9 & 3.95 & 38.4 & -1.28 & 50.4 & -0.8 \\
\hline 0.8 & 44 & 3 & 45.5 & 4.68 & 50.4 & 1.9 \\
\hline
\end{tabular}

mole fraction of [EMIM] [SCN] at $T=298.15 \mathrm{~K}[9-12]$. For $[\mathrm{EMIM}][\mathrm{SCN}](1)+\operatorname{pyridine}(2),[\mathrm{EMIM}][\mathrm{SCN}](1)+$ indoline(2), [EMIM] [SCN](1) + quinoline(2), and $[\mathrm{EMIM}][\mathrm{SCN}](1)+$ thiophene(2) systems, this trend increases linearly from $x_{\mathrm{IL}}=0.3$ to $x_{\mathrm{IL}}=0.8$. [EMIM] [SCN] $(1)+$ thiophene(2) mixture gave higher surface tension because of the similarity in structure of thiophene to [EMIM] cation. The dissimilar structure of pyridine, indoline, and quinoline in [EMIM][SCN] gave higher surface tension as compared to water with $[\mathrm{EMIM}][\mathrm{SCN}]$. It can be seen in Figure 3 that the surface tension increases in the order of thiophene $>$ quinoline $>$ pyridine $>$ indoline $>$ pyrrole $>$ water. Thus an increasing mole fraction of [EMIM] [SCN] causes a decrease in the strength of sterical hindrance due to dissimilar molecules or addition of benzene ring in pyrrole or pyridine structure The van der Waals force between the ethyl substitution on the [EMIM] and the aromatic structure of nitrogen/sulphur is not much significant in liquid phase. Further when the similar or dissimilar aromatic structures of molecules are mixed together, then the interaction between [EMIM] cation and [SCN] anion decreases while the $\mathrm{CH}-\pi$ bond interaction, $\pi-\pi$ stacking, and dispersion forces get increases particularly for the systems having similar or dissimilar aromatic structure of molecules [37].

Figure 4 shows the composition dependence of refractive index for the above-mentioned binary systems (seen in Table 6). Refractive index for [EMIM] [SCN](1) + pyrrole(2) systems decreases linearly with increasing mole fraction ( 0.2 to 0.8$)$ of [EMIM] [SCN] because there is no mobility of ion upon mixing over the entire mole fraction of [EMIM][SCN] at $T=298.15 \mathrm{~K}$. This similar behavior has been observed for other studied systems such as $[$ EMIM] [SCN] (1) + thiophene(2), [EMIM] [SCN] (1) + pyridine(2), $[$ EMIM $][S C N](1)+$ indoline $(2)$, and $[$ EMIM $][S C N](1)+$

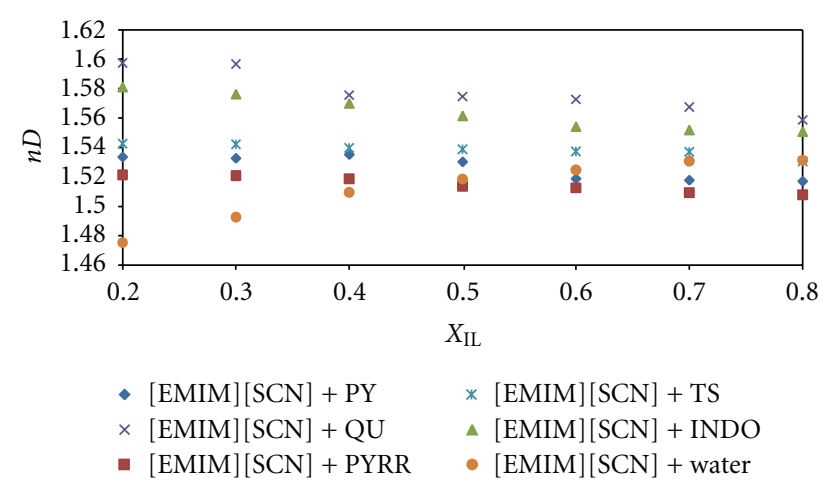

Figure 4: Composition dependence of surface tension of the binary mixture plotted against mole fraction of IL at $298.15 \mathrm{~K}$.

quinoline(2). The only exception is, [EMIM] [SCN](1) + water(2) system which shows an increasing trend with increasing mole fraction due to the increased strength of hydrogen bond. It is clear from Figure 4 that, for the binary mixture under study, the refractive index increases in the order of [EMIM][SCN]: pyrrole < Pyridine < thiophene $<$ indoline $<$ quinoline. Thus [EMIM] $[\mathrm{SCN}]+$ quinoline mixture has the highest refractive index values as compared to other studied systems.

4.5. Effect of Composition on Excess Molar Volume. The excess molar volume can be defined by $[38,39]$

$$
\begin{aligned}
V_{m}^{E} & =\frac{\left(x_{1} M_{1}+x_{2} M_{2}\right)}{\rho_{\text {mix }}}-x_{1} \frac{M_{1}}{\rho_{1}}-x_{2} \frac{M_{2}}{\rho_{2}} \\
& =V_{\text {mix }}-x_{1} V_{1}-x_{2} V_{2},
\end{aligned}
$$


TABLE 6: Experimental refractive index $n D$ and deviation of refractive index $\Delta n D$ for the binary systems: (a) [EMIM] [SCN] + Pyridine, (b) $[$ EMIM $][S C N]+$ Pyrrole, (c) $[$ EMIM $][S C N]+$ Indoline, (d) $[$ EMIM] $[S C N]+$ Quinoline, (e) $[$ EMIM] $[$ SCN $]+$ Thiophene, and (f) $[\mathrm{EMIM}][\mathrm{SCN}]+$ Water at $298.15 \mathrm{~K}$.

\begin{tabular}{|c|c|c|c|c|c|c|}
\hline \multirow{2}{*}{$x_{\mathrm{IL}}$} & \multicolumn{2}{|c|}{ [EMIM] [SCN] + PY } & \multicolumn{2}{|c|}{ [EMIM] [SCN] + PYRR } & \multicolumn{2}{|c|}{$[\mathrm{EMIM}][\mathrm{SCN}]+\mathrm{QU}$} \\
\hline & $n D$ & $\Delta n D$ & $n D$ & $\Delta n D$ & $n D$ & $\Delta n D$ \\
\hline 0.2 & 1.5335 & 0.02492 & 1.5214 & 0.02314 & 1.5974 & 0.0901 \\
\hline 0.3 & 1.5326 & 0.02038 & 1.5209 & 0.01771 & 1.5968 & 0.0863 \\
\hline 0.4 & 1.5351 & 0.01924 & 1.5187 & 0.01058 & 1.5756 & 0.0819 \\
\hline 0.5 & 1.5302 & 0.0107 & 1.5137 & 0.00065 & 1.5746 & 0.0569 \\
\hline 0.6 & 1.5188 & -0.00434 & 1.5126 & -0.00538 & 1.5727 & 0.0521 \\
\hline 0.7 & 1.5178 & -0.00898 & 1.5093 & -0.01361 & 1.5675 & 0.0464 \\
\hline 0.8 & 1.5171 & -0.01332 & 1.5079 & -0.01994 & 1.5587 & 0.0374 \\
\hline \multirow{2}{*}{$x_{\mathrm{IL}}$} & \multicolumn{2}{|c|}{ [EMIM] [SCN] + INDO } & \multicolumn{2}{|c|}{$[$ EMIM] $[\mathrm{SCN}]+\mathrm{TS}$} & \multicolumn{2}{|c|}{ [EMIM] [SCN] + WATER } \\
\hline & $n D$ & $\Delta n D$ & $n D$ & $\Delta n D$ & $n D$ & $\Delta n D$ \\
\hline 0.2 & 1.581 & 0.0041 & 1.5426 & 0.01338 & 1.4754 & 0.11186 \\
\hline 0.3 & 1.5762 & 0.0042 & 1.5421 & 0.01182 & 1.4927 & 0.10739 \\
\hline 0.4 & 1.5699 & 0.0028 & 1.5398 & 0.00846 & 1.5095 & 0.10242 \\
\hline 0.5 & 1.5614 & -0.0008 & 1.5388 & 0.0064 & 1.5186 & 0.08975 \\
\hline 0.6 & 1.5541 & -0.0032 & 1.5373 & 0.00384 & 1.5247 & 0.07408 \\
\hline 0.7 & 1.5518 & -0.0006 & 1.537 & 0.00248 & 1.5308 & 0.05841 \\
\hline 0.8 & 1.5507 & 0.0032 & 1.5305 & -0.00508 & 1.5314 & 0.03724 \\
\hline
\end{tabular}

where $V$ is the molar volume of the pure fluid, $\rho$ is the density of the pure fluid, and subscript $P$ indicates constant pressure. $V_{m}^{E}$ is the excess molar volume of the binary mixture, $\rho_{\text {mix }}$ is density of the mixture, and density of the pure components is denoted as $\rho_{1}$ and $\rho_{2}$, respectively. $x_{1}$ and $x_{2}$ are the mole fraction of components 1 and 2. $M_{1}, M_{2}$ are the molecular weight of components 1 and 2, respectively.

The calculated excess molar volume values are listed in Tables 4(a) to 4(f). [EMIM][SCN](1) + pyridine(2) (Figure 5) binary mixture exhibits a linear decrease with an increase in the mole fraction of [EMIM][SCN](1) at all studied temperatures. At temperature $T=298.15 \mathrm{~K}$ a negative excess molar volume for all the systems is obtained within 0.2 to 0.8 mole fraction. This is due to fact that the specific interactions include the partial charge transfer, formation of hydrogen bond, $\mathrm{CH}-\pi$ bond, $\pi-\pi$ stacking, and $n-\pi$ bond interaction. The weak H-bonds are easily broken up when the temperature increases as compared to $\mathrm{CH}-\pi$ bond, $\pi-\pi$ stacking, and $n-\pi$ bond. This indicates that the IL-sulphur/nitrogen mixture has stronger interaction as compared to IL-IL, thiophene-thiophene, or pyrrole-pyrrole interaction. A similar phenomenon is applicable for all other studied systems. This can also be explained by the stronger association occurring between similar or dissimilar aromatic structure of molecules in liquid phase at any composition or temperatures without any temperature and composition influence.

In case of [EMIM] [SCN](1) + water(2) mixture, a sudden decrease is seen at initial mole fraction, thereafter decreasing linearly as the composition increases. This is due to the fact that at individual concentration (higher dilution rate), the oxygen sites are stabilized by electron or partial charge transfer. Thus [EMIM][SCN] has strong attraction with water at low concentrations. Furthermore the temperature effect on the excess molar volume is negligible since the rate of kinetic energy is not enough to change the phase behavior upon mixing with aromatic structure of the nitrogen/sulphur molecules. It means that the structural effect becomes more important than the dispersion or dipolar interactions. Hence the composition has significant influence on excess molar volume of aromatic structure with multiple ring compounds like indoline and quinoline.

4.6. Effect of Composition on Deviation of Surface Tension and Refractive Index. The deviation of surface tension and refractive index can be defined by [35-39]

$$
\begin{gathered}
\Delta \sigma=\sigma_{\text {mix }}-\left(\sigma_{1} x_{1}-\sigma_{2} x_{2}\right) \\
\Delta n D=n D_{\text {mix }}-\left(n D_{1} x_{1}-n D_{2} x_{2}\right),
\end{gathered}
$$

where $\Delta \sigma$ and $\Delta n D$ are the deviation of surface tension and refractive index. The surface tension and refractive index of the binary mixture are denoted as $\sigma_{\text {mix }}$ and $n D_{\text {mix }}$, respectively. $x_{1}$ and $x_{2}$ are the mole fraction of the components 1 and 2 .

The deviation of surface tension and refractive index are presented in Figures 6 and 7. The deviation of surface tension for $[\mathrm{EMIM}][\mathrm{SCN}](1)+\operatorname{pyrrole}(2)$ indicates an increasing trend with increasing mole fraction of [EMIM] [SCN] while the refractive index deviation shows a decreasing trend at $T=298.15 \mathrm{~K}$. It can be observed that the strong electrostatic interaction takes place between the two similar aromatic structures of the molecules (IL-nitrogen/sulphur compound), even though molecules posses additional benzene ring such as indoline and quinoline. The deviation of surface tension was found to be inversely proportional to deviation of refractive index. This is due to the fact 


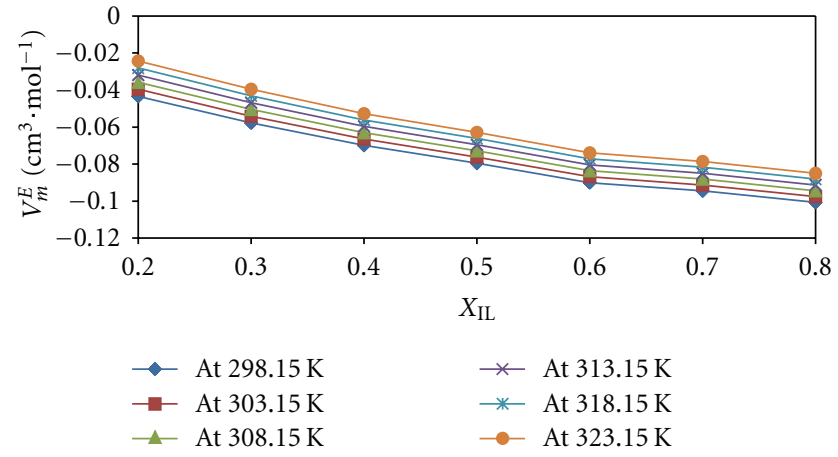

Figure 5: Excess molar volume $V_{m}^{E}$ for the system of $[\mathrm{EMIM}][\mathrm{SCN}](1)+$ pyridine(2) at different temperatures.

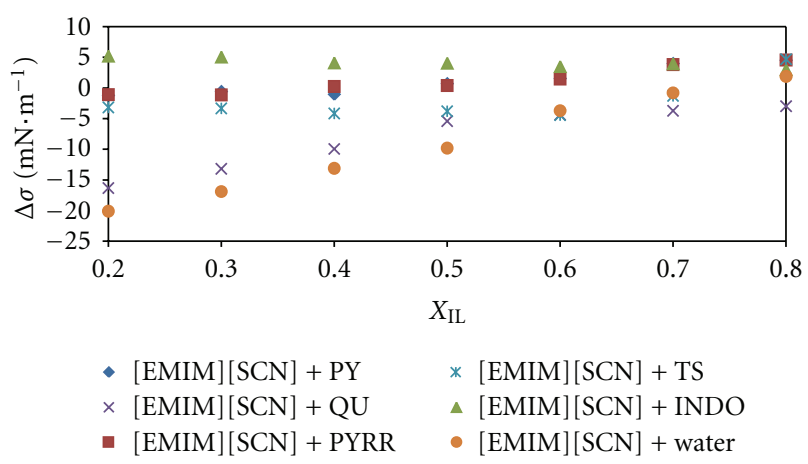

Figure 6: Surface tension deviation versus mole fraction of IL at $298.15 \mathrm{~K}$.

that the deviation of surface tension of the binary mixture strongly depends on the cohesive interaction at the surface level while the refractive index deviation depends on the dispersion interaction upon mixing. However both depend on the size and shape of the molecules in a liquid phase. These tendencies agree well with the reported data of Nain [40] and Freire et al. [41]. In [EMIM][SCN] + Water mixture, the deviation of surface tension increases linearly with composition (Figure 7) while the deviation of refractive index decreases slightly (Figure 8). The molecular interaction is very strong between similar and dissimilar aromatic structures [31, 34, 42, 43] of sulphur/nitrogen compounds and [EMIM] cation while the mobility of ions is weakened upon mixing. However [EMIM][SCN] interacts with the compounds in liquid phase via $n-\pi$ bonding (where $n$ : $\mathrm{N}, \mathrm{O}$ from both the side). It can be observed that the minimum amount of [EMIM] [SCN] ionic liquid is enough for separation of similar structure of pyrrole and thiophene while for pyridine, indoline, and quinoline more amount is required as compared to pyrrole and thiophene. Negative deviation was observed for the mole fraction ( 0.2 to 0.8$)$ of IL at temperature from 298.15 to $323.15 \mathrm{~K}$ for both deviation in surface tension and refractive index studies. The observed trends are as follows.

For surface tension deviation:

Water $>$ quinoline $>$ thiophene $>$ pyrrole $>$ pyridine $>$ indoline.

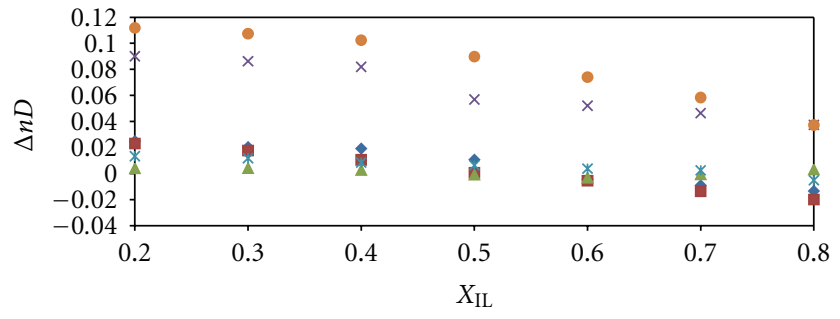

$$
\begin{array}{rlr} 
& {[\mathrm{EMIM}][\mathrm{SCN}]+\mathrm{PY}} & *[\mathrm{EMIM}][\mathrm{SCN}]+\mathrm{TS} \\
\times[\mathrm{EMIM}][\mathrm{SCN}]+\mathrm{QU} & \Delta[\mathrm{EMIM}][\mathrm{SCN}]+\mathrm{INDO} \\
- & {[\mathrm{EMIM}][\mathrm{SCN}]+\mathrm{PYRR}} & \bullet[\mathrm{EMIM}][\mathrm{SCN}]+\text { water }
\end{array}
$$

FIGURE 7: Refractive index deviation against mole fraction of IL at $298.15 \mathrm{~K}$.

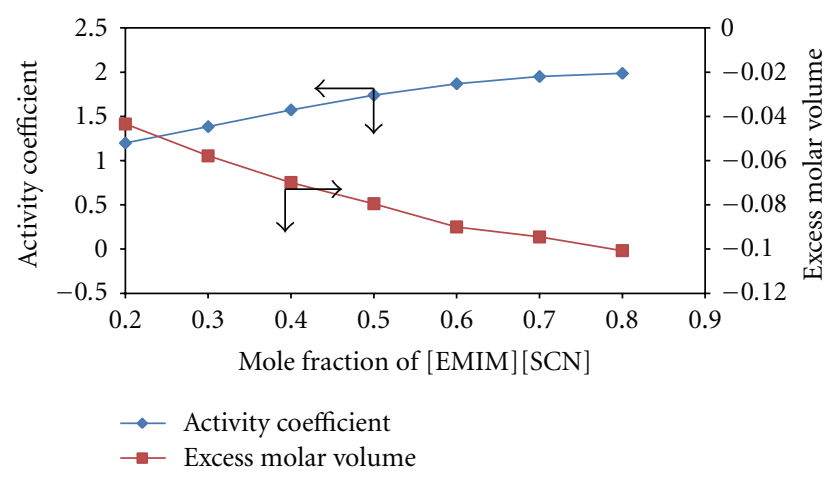

FIGURE 8: Relation of activity coefficient with excess molar volume of [EMIM] [SCN](1) + pyridine(2) systems at $T=298.15 \mathrm{~K}$.

For refractive index deviation:

Indoline $>$ thiophene $>$ pyrrole $>$ pyridine $>$ quinoline $>$ water.

4.7. Relation of Activity Coefficient with Excess Molar Volume. In order to substantiate the findings we have predicted the activity coefficients of solutes (nitrogen and sulphur compounds) in mixture (Tables $4(\mathrm{a})$ to $4(\mathrm{f})$ ). It is interesting to know that that pyrrole (Table 4(b)) and water (Table 4(c)) have activity coefficients less than one among all the studied systems. It implies that water is soluble in IL, thus the IL is hydrophilic in nature. Among all the compounds it seems that pyrrole is the most soluble. Figure 8 shows a comparison of predicted activity coefficient and excess volume for ILpyridine mixture. The predicted activity coefficient increases with increasing mole fraction of ionic liquid in the mixture, except for [EMIM][SCN] + water systems. However the excess molar volumes are seen to decrease with mole fraction. Thus it is fair to say that the activity coefficient is inversely proportional to excess volume.

It is observed that the properties of mixture are influenced by the aromatic ring structure of thiophene, pyridine, pyrrole, indoline, and quinoline (Tables 4(a) to 4(f)). The activity coefficient also gives us an indication of the strength of solubility. For example, [EMIM] [SCN](1) + Pyridine(2) system (Figure 8) shows low activity coefficient (i.e., high solubility with mole fraction of IL) as compared to 
[EMIM] $[\mathrm{SCN}]+$ thiophene (Table 4(e)) which implies that the solubility of pyridine in [EMIM] [SCN] is higher than thiophene. In case of excess molar volume both the systems show an increasing excess molar volume with increasing mole fraction of IL.

\section{Conclusion}

Excess molar volume and deviation of surface tension and refractive index have been calculated from measured densities, surface tension, and refractive index for the systems of [EMIM] [SCN] ionic liquid with pyrrole, pyridine, indoline, quinoline, thaiophene, and water in a liquid phase at temperature of 298.15 to $323.15 \mathrm{~K}$ and atmospheric pressure. The surface tension increases with increasing mole fraction in the order of thiophene $>$ quinoline $>$ pyridine $>$ indoline $>$ pyrrole $>$ water, while the refractive index increases in the order of [EMIM] [SCN]: pyrrole $<$ Pyridine $<$ thiophene $<$ indoline $<$ quinoline. Furthermore the deviation of surface tension and refractive index show a similar negative trend which follows: for surface tension: Water $>$ quinoline $>$ thiophene $>$ pyrrole $>$ pyridine $>$ indoline, for refractive index: Indoline $>$ thiophene $>$ pyrrole $>$ pyridine $>$ quinoline $>$ water. Finally it was concluded that the similar aromatic structures of nitrogen and/or sulphur compounds are removed via $\mathrm{CH}-\pi$ bond interaction and $\pi-\pi$ stacking. The aromatic heterocycle with multiple ring of nitrogen and sulphur compounds removal was found to strongly depend on the composition of IL as compared to temperature effect. It is also observed that the activity coefficient of IL increases while the excess molar volumes decreases with increasing mole fraction of IL.

\section{Abbreviations}

$\begin{array}{ll}\text { HOMO: } & \text { Higher occupied molecular orbitals } \\ \text { LUMO: } & \text { Lower unoccupied molecular orbitals } \\ {[\text { EMIM]: }} & \text { 1-ethyl-3-methylimidazolium } \\ {[\text { SCN]: }} & \text { Thiocyanate } \\ {[\text { EMIM][SCN]: }} & \text { 1-ethyl-3-methylimidazolium } \\ & \text { Thiocyanate } \\ \text { PY: } & \text { Pyridine } \\ \text { PYRR: } & \text { Pyrrole } \\ \text { INDO: } & \text { Indoline } \\ \text { QU: } & \text { Quinoline } \\ \text { TS: } & \text { Thiophene. }\end{array}$

\section{Acknowledgment}

The authors are grateful to the Department of Science and Technology (DST), Government of India for the financial support through Project SR/FTP/08-08 under the Fast Track Scheme.

\section{References}

[1] A. Jayaraman, F. H. Yang, and R. T. Yang, "Effects of nitrogen compounds and polyaromatic hydrocarbons on desulfurization of liquid fuels by adsorption via $\pi$-complexation with
Cu(I)Y zeolite," Energy and Fuels, vol. 20, no. 3, pp. 909-914, 2006.

[2] S. Ramírez, C. Cabrera, C. Aguilar et al., "Two stages light gasoil hydrotreating for low sulfur diesel production," Catalysis Today, vol. 98, no. 1-2, pp. 323-332, 2004.

[3] T. Song, Z. Zhang, J. Chen, Z. Ring, H. Yang, and Y. Zheng, "Effect of aromatics on deep hydrodesulfurization of dibenzothiophene and 4,6-dimethyldibenzothiopene over NiMo/Al2O3 catalyst," Energy and Fuels, vol. 20, no. 6, pp. 2344-2349, 2006.

[4] H. Yang, J. Chen, Y. Briker, R. Szynkarczuk, and Z. Ring, "Effect of nitrogen removal from light cycle oil on the hydrodesulphurization of dibenzothiophene, 4-methyldibenzothiophene and 4,6-dimethyldibenzothiophene," Catalysis Today, vol. 109, no. 1-4, pp. 16-23, 2005.

[5] H. J. Jeon, C. H. Ko, S. H. Kim, and J. N. Kim, "Removal of refractory sulfur compounds in diesel using activated carbon with controlled porosity," Energy and Fuels, vol. 23, no. 5, pp. 2537-2543, 2009.

[6] J. H. Shan, X. Q. Liu, L. B. Sun, and R. Cui, "Cu-Ce bimetal ion-exchanged $Y$ zeolites for selective adsorption of thiophenic sulfur," Energy and Fuels, vol. 22, no. 6, pp. 3955-3959, 2008.

[7] H. Gao, Y. Li, Y. Wu et al., "Extractive desulfurization of fuel using 3-methylpyridinium-based ionic liquids," Energy and Fuels, vol. 23, no. 5, pp. 2690-2694, 2009.

[8] Y. Nie, C. Li, A. Sun, H. Meng, and Z. Wang, "Extractive desulfurization of gasoline using imidazolium-based phosphoric ionic liquids," Energy and Fuels, vol. 20, no. 5, pp. 2083-2087, 2006.

[9] S. Zhang, Q. Zhang, and Z. C. Zhang, "Extractive desulfurization and denitrogenation of fuels using ionic liquids," Industrial and Engineering Chemistry Research, vol. 43, no. 2, pp. 614-622, 2004.

[10] S. Zhang and Z. C. Zhang, "Novel properties of ionic liquids in selective sulfur removal from fuels at room temperature," Green Chemistry, vol. 4, no. 4, pp. 376-379, 2002.

[11] X. Jiang, Y. Nie, C. Li, and Z. Wang, "Imidazolium-based alkylphosphate ionic liquids - a potential solvent for extractive desulfurization of fuel," Fuel, vol. 87, no. 1, pp. 79-84, 2008.

[12] R. Anantharaj and T. Banerjee, "Quantum chemical studies on the simultaneous interaction of thiophene and pyridine with ionic liquid," AIChE Journal, vol. 57, no. 3, pp. 749-764, 2011.

[13] R. Anantharaj and T. Banerjee, "COSMO-RS-based screening of ionic liquids as green solvents in denitrification studies," Industrial and Engineering Chemistry Research, vol. 49, no. 18, pp. 8705-8725, 2010.

[14] R. Anantharaj and T. Banerjee, "COSMO-RS based predictions for the desulphurization of diesel oil using ionic liquids: effect of cation and anion combination," Fuel Processing Technology, vol. 92, no. 1, pp. 39-52, 2011.

[15] N. R. Varma, A. Ramalingam, and T. Banerjee, "Experiments, correlations and COSMO-RS predictions for the extraction of benzothiophene from n-hexane using imidazolium-based ionic liquids," Chemical Engineering Journal, vol. 166, no. 1, pp. 30-39, 2011.

[16] S. P. Singh, R. Anantharaj, and T. Banerjee, "UNIFAC group interaction prediction for ionic liquid-thiophene based systems using genetic algorithm," Lecture Notes in Computer Science, vol. 6457, pp. 195-204, 2010.

[17] R. Anantharaj and T. Banerjee, "Evaluation and comparison of global scalar properties for the simultaneous interaction of ionic liquids with thiophene and pyridine," Fluid Phase Equilibria, vol. 293, no. 1, pp. 22-31, 2010. 
[18] A. A. P. Kumar and T. Banerjee, "Thiophene separation with ionic liquids for desulphurization: a quantum chemical approach," Fluid Phase Equilibria, vol. 278, no. 1-2, pp. 1-8, 2009.

[19] U. Domańska, M. Królikowska, and M. Królikowski, "Phase behaviour and physico-chemical properties of the binary systems 1-ethyl-3-methylimidazolium thiocyanate, or 1-ethyl-3methylimidazolium tosylate + water, or + an alcohol," Fluid Phase Equilibria, vol. 294, no. 1-2, pp. 72-83, 2010.

[20] Y. Geng, T. Wang, D. Yu, C. Peng, H. Liu, and Y. Hu, "Densities and Viscosities of the Ionic Liquid [C4mim] [PF6]+ N, N-dimethylformamide Binary Mixtures at $293.15 \mathrm{~K}$ to $318.15 \mathrm{~K} *$," Chinese Journal of Chemical Engineering, vol. 16, no. 2, pp. 256-262, 2008.

[21] E. Sapei, A. Zaytseva, P. Uusi-Kyyny, K. I. Keskinen, and J. Aittamaa, "Vapor-liquid equilibrium for binary system of thiophene + n-hexane at (338.15 and 323.15) $\mathrm{K}$ and thiophene + 1-hexene at (333.15 and 323.15) K," Journal of Chemical and Engineering Data, vol. 51, no. 6, pp. 2203-2208, 2006.

[22] S. Erlin, A. Zaytseva, P. Uusi-Kyyny, K. Younghun, K. I. Keskinen, and J. Aittamaa, "Vapor-liquid equilibrium for binary system of 1-propanethiol, thiophene, and diethyl sulfide with toluene at $90.03 \mathrm{kPa}$," Journal of Chemical and Engineering Data, vol. 51, no. 4, pp. 1372-1376, 2006.

[23] E. Gómez, B. González, N. Calvar, E. Tojo, and A. Domínguez, "Physical properties of pure 1-ethyl-3-methylimidazolium ethylsulfate and its binary mixtures with ethanol and water at several temperatures," Journal of Chemical and Engineering Data, vol. 51, no. 6, pp. 2096-2102, 2006.

[24] H. Rodríguez and J. F. Brennecke, "Temperature and composition dependence of the density and viscosity of binary mixtures of water + ionic liquid," Journal of Chemical and Engineering Data, vol. 51, no. 6, pp. 2145-2155, 2006.

[25] A. Wandschneider, J. K. Lehmann, and A. Heintz, "Surface tension and density of pure ionic liquids and some binary mixtures with 1-propanol and 1-butanol," Journal of Chemical and Engineering Data, vol. 53, no. 2, pp. 596-599, 2008.

[26] A. B. Pereiro, E. Tojo, A. Rodríguez, J. Canosa, and J. Tojo, "Properties of ionic liquid HMIMPF6 with carbonates, ketones and alkyl acetates," Journal of Chemical Thermodynamics, vol. 38, no. 6, pp. 651-661, 2006.

[27] A. B. Pereiro, J. L. Legido, and A. Rodríguez, "Physical properties of ionic liquids based on 1-alkyl-3-methylimidazolium cation and hexafluorophosphate as anion and temperature dependence," Journal of Chemical Thermodynamics, vol. 39, no. 8, pp. 1168-1175, 2007.

[28] U. Domańska, Z. Zołek-Tryznowska, and M. Królikowski, "Thermodynamic phase behavior of ionic liquids," Journal of Chemical and Engineering Data, vol. 52, no. 5, pp. 1872-1880, 2007.

[29] U. Domańska and M. Laskowska, "Phase equilibria and volumetric properties of (1-ethyl-3-methylimidazolium ethylsulfate+alcohol or water) binary systems," Journal of Solution Chemistry, vol. 37, no. 9, pp. 1271-1287, 2008.

[30] D. B. Won, S. J. Park, K. J. Han, and C. J. Kim, "Liquid-liquid equilibria for methanol + hexadecane + heterocyclic nitrogencontaining compounds at 298.15 K," Fluid Phase Equilibria, vol. 193, no. 1-2, pp. 217-227, 2002.

[31] P. Acharya, O. Plashkevych, C. Morita, S. Yamada, and J. Chattopadhyaya, "A repertoire of pyridinium-phenyl-methyl cross-talk through a cascade of intramolecular electrostatic interactions," Journal of Organic Chemistry, vol. 68, no. 4, pp. 1529-1538, 2003.
[32] H. Suezawa, T. Hashimoto, K. Tsuchinaga et al., "Electronic substituent effect on intramolecular $\mathrm{CH} / \pi$ interaction as evidenced by NOE experiments," Journal of the Chemical Society. Perkin Transactions, vol. 2, no. 6, pp. 1243-1249, 2000.

[33] H. Suezawa, S. Ishihara, Y. Umezawa, S. Tsuboyama, and M. Nishio, "The aromatic $\mathrm{CH} / \pi$ hydrogen bond as an important factor in determining the relative stability of diastereomeric salts relevant to enantiomeric resolution-a crystallographic database study," European Journal of Organic Chemistry, no. 23, pp. 4816-4822, 2004.

[34] J. A. Joule and K. Mills, K.Heterocyclic Chemistry, Blackwell, Oxford, UK, 4th edition, 2007.

[35] R. R. Gupta, M. Kumar, and V. Gupta, Heterocyclic Chemistry-II; Five-Membered Heterocyclic, Springer, New York, NY, USA, 2005.

[36] R. K. Bansal, Heterocyclic Chemistry, New Age International, New Delhi, India, 4th edition, 2005.

[37] M. T. Zafarani-Moattar and R. Majdan-Cegincara, "Viscosity, density, speed of sound, and refractive index of binary mixtures of organic solvent + Ionic liquid, 1-Butyl-3-methylimidazolium hexafluorophosphate at $298.15 \mathrm{~K}$," Journal of Chemical and Engineering Data, vol. 52, no. 6, pp. 2359-2364, 2007.

[38] A. Arce, E. Rodil, and A. Soto, "Volumetric and viscosity study for the mixtures of 2-ethoxy-2- methylpropane, ethanol, and 1-ethyl-3-methylimidazolium ethyl sulfate ionic liquid," Journal of Chemical and Engineering Data, vol. 51, no. 4, pp. 1453-1457, 2006.

[39] J. M. S. S. Esperança, H. J. R. Guedes, M. Blesic, and L. P. N. Rebelo, "Densities and derived thermodynamic properties of ionic liquids. 3. Phosphonium-based ionic liquids over an extended pressure range," Journal of Chemical and Engineering Data, vol. 51, no. 1, pp. 237-242, 2006.

[40] A. K. Nain, "Refractive indices and deviations in refractive indices for binary mixtures of formamide +1 -butanol, +2 butanol, + 1,3-butanediol, and + 1,4-butanediol at temperatures from (293.15 to 318.15) K," Journal of Chemical and Engineering Data, vol. 53, no. 5, pp. 1208-1210, 2008.

[41] M. G. Freire, P. J. Carvalho, A. M. Fernandes, I. M. Marrucho, A. J. Queimada, and J. A. P. Coutinho, "Surface tensions of imidazolium based ionic liquids: anion, cation, temperature and water effect," Journal of Colloid and Interface Science, vol. 314, no. 2, pp. 621-630, 2007.

[42] Q. Zhou, L. S. Wang, and H. P. Chen, "Densities and viscosities of 1-Butyl-3-methylimidazolium tetrafluoroborate $+\mathrm{H} 2 \mathrm{O}$ binary mixtures from (303.15 to 353.15) K," Journal of Chemical and Engineering Data, vol. 51, no. 3, pp. 905-908, 2006.

[43] S. Zhang, X. Li, H. Chen, J. Wang, J. Zhang, and M. Zhang, "Determination of physical properties for the binary system of 1-ethyl-3-methylimidazolium tetrafluoroborate + $\mathrm{H} 2 \mathrm{O}$," Journal of Chemical and Engineering Data, vol. 49, no. 4, pp. 760-764, 2004. 

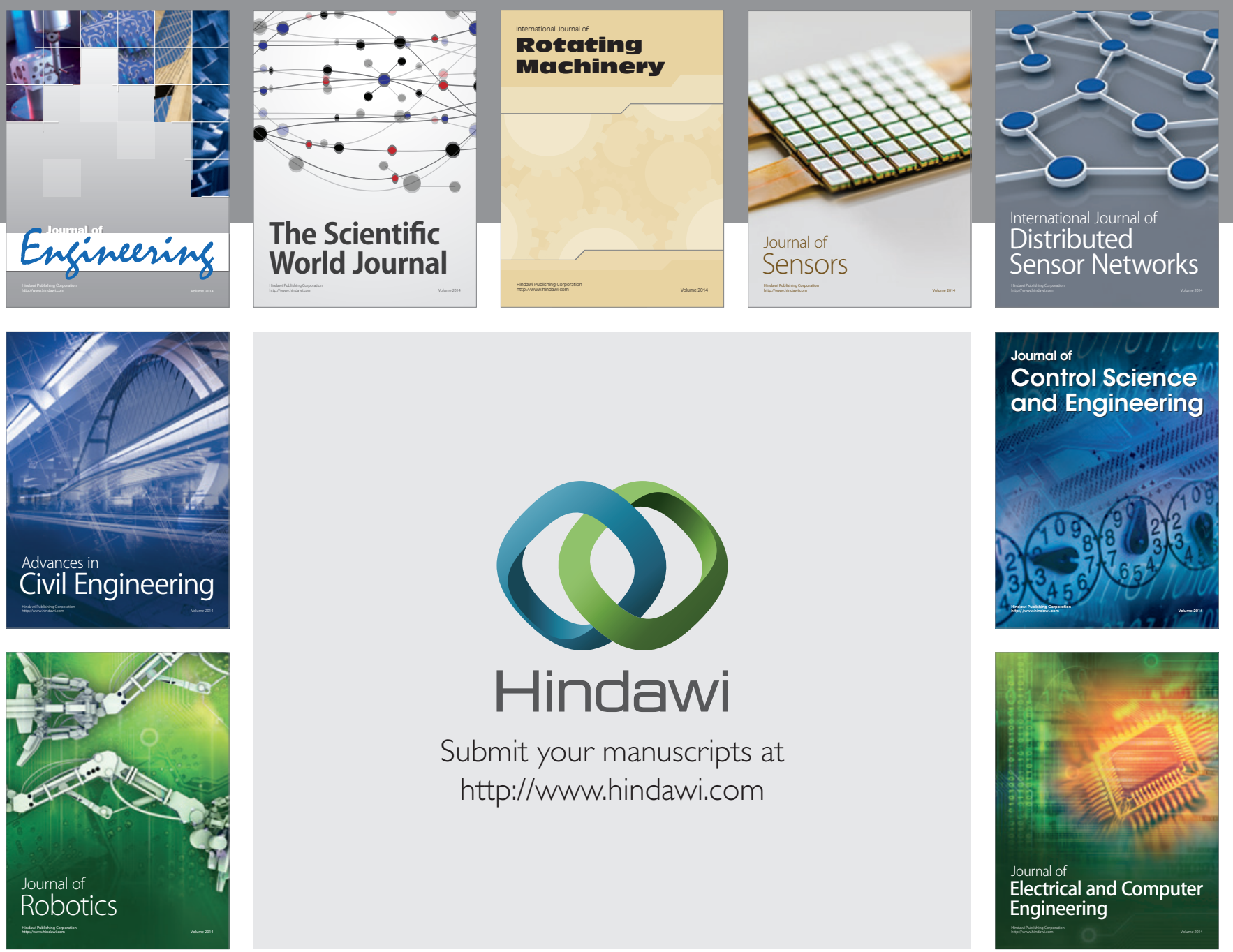

Submit your manuscripts at

http://www.hindawi.com
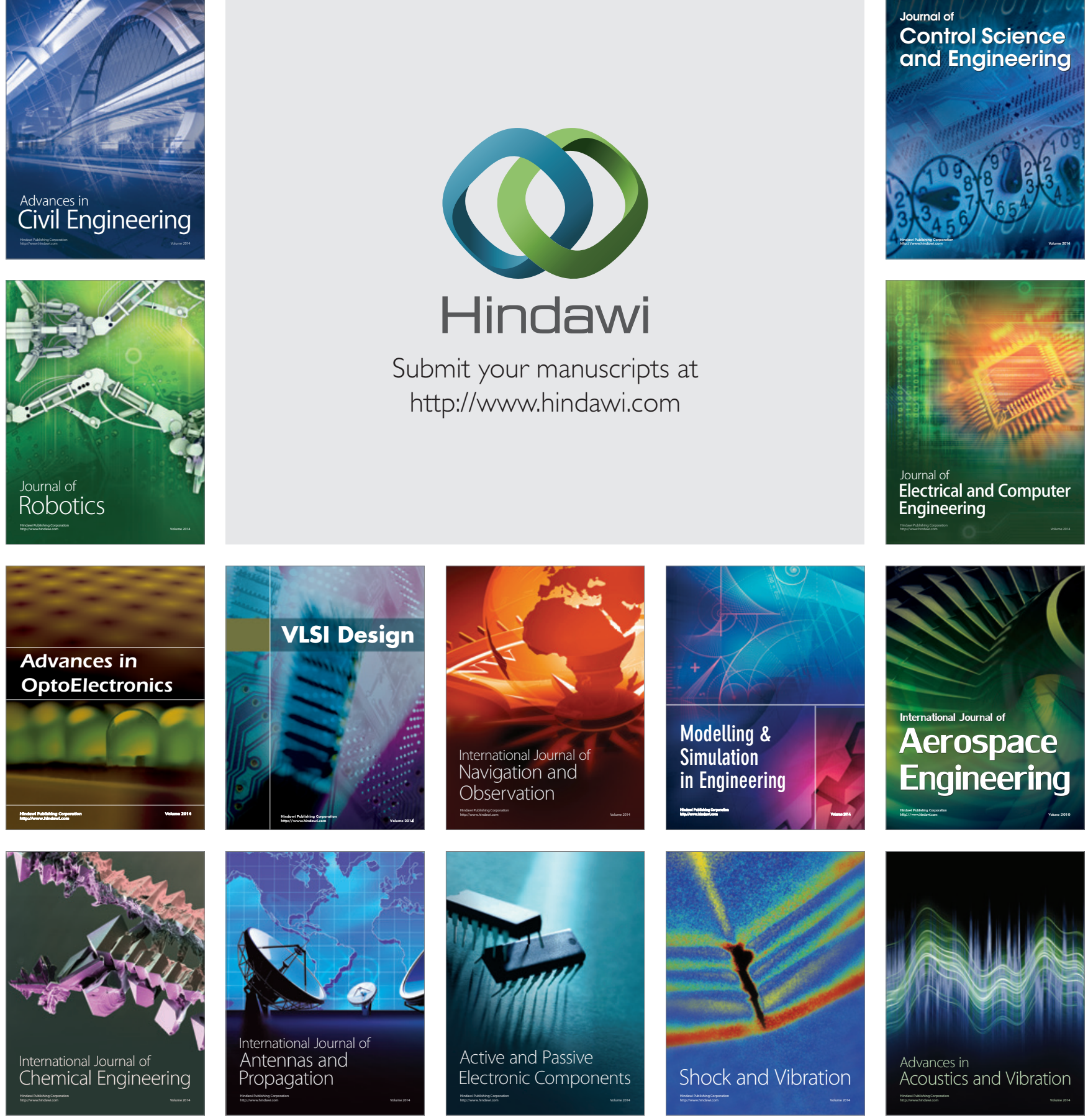